\title{
SOLUTION OF MONOTONE COMPLEMENTARITY AND GENERAL CONVEX PROGRAMMING PROBLEMS USING A MODIFIED POTENTIAL REDUCTION INTERIOR POINT METHOD*
}

\author{
KUO-LING HUANG AND SANJAY MEHROTRA ${ }^{\dagger}$
}

\begin{abstract}
We present a homogeneous algorithm equipped with a modified potential function for the monotone complementarity problem. We show that this potential function is reduced by at least a constant amount if a scaled Lipschitz condition is satisfied. A practical algorithm based on this potential function is implemented in a software package named iOptimize. The implementation in iOptimize maintains global linear polynomial-time convergence properties while achieving practical performance. When compared with a mature software package MOSEK (barrier solver version 6.0.0.106), iOptimize solves convex quadratic programming problems, convex quadratically constrained quadratic programming problems, and general convex programming problems in fewer iterations. Moreover, several problems for which MOSEK fails are solved to optimality. We also find that iOptimize seems to detect infeasibility more reliably than general nonlinear solvers Ipopt (version 3.9.2) and Knitro (version 8.0).
\end{abstract}

Key words. quadratic programs, quadratically constrained quadratic programs, convex programs, homogeneous algorithms, interior point methods

AMS subject classifications. 90C06, 90C20, 90C25, 90C30, 90C33, 90C51, 49M15

1. Introduction. In this paper we consider a monotone complementarity problem (MCP) of the following form

$$
\begin{gathered}
s=f(x) \\
0 \leq s \perp x \geq 0,
\end{gathered}
$$

where $x, s \in R^{n}, f(x)$ is a continuous monotone mapping from $R_{+}^{n}:=\left\{x \in R^{n} \mid x \geq 0\right\}$ to $R^{n}$, and the notation $0 \leq s \perp x \geq 0$ means that $(x, s) \geq 0$ and that $x^{T} s=0$. The requirement $x^{T} s=0$ is called complementarity condition. Then, for every $x^{1}, x^{2} \in R_{+}^{n}$, we have

$$
\left(x^{1}-x^{2}\right)^{T}\left(f\left(x^{1}\right)-f\left(x^{2}\right)\right) \geq 0 .
$$

Let $\nabla f(x)$ denote the Jacobian matrix of $f(x)$. If $\nabla f(x)$ is positive semidefinite for all $x>0$, that is,

$$
h^{T} \nabla f(x) h \geq 0, \forall x>0, h \in R^{n},
$$

then $f(x)$ is a continuous monotone mapping.

$(x, s) \subset R_{+}^{2 n}$ is called a feasible solution of MCP if (1.1) is satisfied. It is called an optimal or complementary solution if both (1.1) and (1.2) are satisfied. If MCP has a complementary solution $\left(x^{*}, s^{*}\right)$, then it has a maximal complementary solution where the number of positive components of $\left(x^{*}, s^{*}\right)$ is maximal.

Given an initial point $\left(x^{0}, s^{0}\right) \subset R_{++}^{2 n}:=\left\{x \in R^{n} \mid x>0\right\}$, we would like to compute a bounded sequence $\left\langle x^{k}, s^{k}\right\rangle \subset R_{++}^{2 n}, k=0,1, \ldots$, such that

$$
\lim _{k \rightarrow \infty} s^{k}-f\left(x^{k}\right) \rightarrow 0, \text { and } \lim _{k \rightarrow \infty}\left(x^{k}\right)^{T} s^{k} \rightarrow 0 .
$$

${ }^{*}$ The research of both authors was partially supported by grant DOE-SP0011568 and ONR N00014-09-10518, N00014-210051

${ }^{\dagger}$ Dept. of Industrial Engineering and Management Sciences, Northwestern University, Evanston, IL, 60208, Email: mehrotra@iems.northwestern.edu 
Let $X:=\operatorname{diag}\left(x_{1}, \ldots, x_{n}\right)$, and let

$$
\nu_{f}(\cdot):(0,1) \mapsto(1, \infty)
$$

be a monotone increasing function. We say that $f$ satisfies a scaled Lipschitz condition (SLC) if

$$
\|X(f(x+h)-f(x)-\nabla f(x) h)\|_{1} \leq \nu_{f}(\beta) h^{T} \nabla f(x) h
$$

whenever

$$
h \in R^{n}, x \in R_{++}^{n},\left\|X^{-1} h\right\|_{\infty} \leq \beta<1,
$$

where $\beta \in(0,1)$ is a constant. The analysis in this paper assumes that $f$ satisfies SLC. Such a condition for MCP was used by Potra and Ye [31] to develop an interior point method (IPM) for MCP. In their analysis Potra and Ye assumed that the set of strictly feasible points $\left\{x \in R^{n} \mid x>0, f(x)>0\right\}$ is nonempty, and a feasible starting point $x^{0}>0$ and $s^{0}=f\left(x^{0}\right)>0$ is available. Under these assumptions Potra and Ye [31] developed an IPM that achieves a constant reduction of the primal-dual potential function

$$
\phi_{\rho}(x, s)=\rho \log x^{T} s-\sum_{j=1}^{n} \log x_{j} s_{j},
$$

for $n+\sqrt{n} \leq \rho \leq 2 n$.

This type of potential function was introduced by Tanabe [35] and Todd and Ye [36] for LP and further extended for linear complementarity problems by Kojima et al. [22]. It satisfies the following inequality

$$
(\rho-n) \log x^{T} s \leq \phi_{\rho}(x, s)-n \log n,
$$

and hence it follows that

$$
x^{T} s \leq \epsilon \text { whenever } \phi_{\rho}(x, s) \leq n \log n-(\rho-n)|\log \epsilon| .
$$

Therefore, starting from a feasible point, if this potential function is reduced by at least a positive constant at each interior point iteration, then we will have $x^{T} s \leq \epsilon$ after at most $\mathcal{O}\left(\phi_{\rho}\left(x^{0}, s^{0}\right)-n \log n+(\rho-n)|\log \epsilon|\right)$ iterations, where $\epsilon$ is the complementarity error.

However, in practice an initial feasible point $x^{0}$ for optimization problems may not be available. To handle this issue, Ye et al. [39] developed a homogeneous IPM for linear programming (LP) that can start from any (infeasible) interior point and achieves the best known convergence complexity without introducing a big M constant. Moreover, it detects the infeasibility of problems unambiguously. Xu et al. [38] developed a simplified (and equivalent) version of the homogeneous IPM, and showed that with similar computational efforts, their method can be more reliable than conventional primal-dual IPM while solving infeasible problems in Netlib [8] LP benchmark library.

Andersen et al. [10] implemented a homogeneous IPM for solving second order conic programming problems based on the work of Nesterov and Todd [28]. Luo [23], Sturm [34] and Zhang [40] extended the homogeneous IPM to general conic programming, including semidefinite programming. Recently, Skajaa et al. [33] implemented a homogeneous IPM for solving nonsymmetric conic programming problems. 
Andersen and Ye $[11,12]$ extended the homogeneous IPM to the general MCP. They developed a homogeneous IPM that converges to a maximal complementary solution in at most $\mathcal{O}(\sqrt{n} \log 1 / \epsilon)$ iterations if $f$ satisfies SLC. Although the global linear polynomial time convergence is provided in their analysis, exact search directions and restrictive step size computations are required, and hence their algorithm is not practical.

Motivated by the apparent gap between the theoretical complexity results and long-step practical implementations in IPM, Mehrotra and Huang [26] recently introduced a homogeneous IPM equipped with a continuously differentiable potential function for LP. Their method ensures a global linear polynomial time convergence while providing the flexibility to integrate heuristics for generating the search directions and the step size computations. More importantly, they showed that it is possible to implement a potential reduction IPM that uses similar number of iterations as an algorithm that ignores global linear polynomial time convergence.

In this paper the Mehrotra-Huang potential function is extended to the context of MCP. Our goal is to develop a stable interior point solver that ensures the global linear polynomial time convergence without compromising the computational efficiency. Two guiding principles are used in our implementation: (1) ensure a reduction in the potential function; (2) use the Mehrotra "corrector" direction to improve the reduction in the potential function. Our algorithm is implemented in a software package named iOptimize. iOptimize is developed based on a variant of Mehrotra's predictor-corrector framework [25]. Computational results on the standard test problems $[2,5,6,24]$ show that iOptimize appears to perform more robustly and solves problems with fewer average iterations than a mature commercial solver MOSEK (barrier solver version 6.0.0.106; MOSEK for short) [7]. In particular, all problems in test sets $[2,5,6,24]$ were solved by iOptimize using default settings, while MOSEK failed on several problems. Moreover, the average number of iterations used by iOptimize are fewer than those taken by MOSEK for convex quadratic programming (QP) problems, convex quadratically constrained quadratic programming (QCQP) problems, and general convex programming $(\mathrm{CP})$ problems in the test sets. We further created some infeasible problems to check the robustness of our approach. While iOptimize solved all these problems (except one, where the failure was due to an AMPL error message), MOSEK failed on several problems. We also found that iOptimize seems to detect infeasibility more reliably than general nonlinear solvers Ipopt (version 3.9.2) [3] and Knitro (version 8.0) [4].

The rest of this paper is organized as follows. In Section 2 we outline the known results for the homogeneous formulation for MCP. In Section 3 we describe our potential function and show that our potential reduction algorithm converges to a maximal complementary solution in polynomial time. In Section 4 we provide additional implementation details of iOptimize. Computational results are presented and discussed in Section 5. Concluding remarks are made in Section 6.

2. Homogeneous formulation for the monotone complementarity problem. In this section we summarize the main results of Andersen and Ye's [11, 12, Theorem 1] homogeneous IPM for MCP. Andersen and Ye consider an augmented homogeneous model related to MCP (HMCP):

$$
\begin{aligned}
& s=\tau f(x / \tau), \\
& \kappa=-x^{T} f(x / \tau),
\end{aligned}
$$




$$
0 \leq(s, \kappa) \perp(x, \tau) \geq 0 .
$$

The right-hand side in (2.1) is closely related to the recession function in convex analysis of Rockafellar [32, Section 8]. Given a maximal complementary solution $\left(x^{*}, \tau^{*}, s^{*}, \kappa^{*}\right)$ that satisfies (2.1) and (2.2), suppose $\tau^{*}>0$ and hence $\kappa^{*}=0$. Then from (2.1) and (2.2) we have

$$
s^{*} / \tau^{*}=f\left(x^{*} / \tau^{*}\right) \text { and } \kappa^{*} / \tau^{*}=-\left(x^{*} / \tau^{*}\right)^{T}\left(s^{*} / \tau^{*}\right)=0 .
$$

This implies that $\left(x^{*} / \tau^{*}, s^{*} / \tau^{*}\right)$ is a complementary solution for MCP. On the other hand, if $\kappa^{*}>0$ and hence $\tau^{*}=0$, then the complementarity of MCP at $\left(x^{*}, s^{*}\right)$ is not zero. In this case MCP is said to be infeasible, and $x^{*}$ is a direction of a separating hyperplane that separates 0 and the set $\left\{s-f(x) \in R^{n} \mid(x, s)>0\right\}$.

Let

$$
F(x, \tau):=\left(\begin{array}{c}
\tau f(x / \tau) \\
-x^{T} f(x / \tau)
\end{array}\right): R_{++}^{n+1} \rightarrow R^{n+1} .
$$

Andersen and Ye [12] showed the following theorem.

THEOREM 2.1. Consider the MCP and the associated HMCP.

1. If $\nabla f$ is positive semidefinite in $R_{+}^{n}$, then $\nabla F$ is also positive semidefinite in $R_{++}^{n+1}$.

2. $F$ is a continuous homogeneous function in $R_{++}^{n+1}$ with degree 1 and for any $(x, \tau) \in R_{++}^{n+1},(x ; \tau)^{T} F(x, \tau)=0$, and $(x, \tau)^{T} \nabla F(x, \tau)=-F(x, \tau)^{T}$.

3. If $f$ is a continuous monotone mapping from $R_{+}^{n}$ to $R^{n}$, then $F$ is a continuous monotone mapping from $R_{++}^{n+1}$ to $R^{n+1}$.

4. If $f$ is scaled Lipschitz with $\nu_{f}$, then $F$ is also scaled Lipschitz; that is, it satisfies condition (1.6) with

$$
\nu_{F}(\beta)=\left(1+\frac{2 \nu_{f}(2 \beta /(1-\beta))}{1-\beta}\right)\left(\frac{1}{1-\beta}\right),
$$

where $\beta<1 / 3$.

5. There exists a bounded sequence $\left\langle x^{k}, \tau^{k}, s^{k}, \kappa^{k}\right\rangle \subset R_{++}^{2 n+2}, k=0,1, \ldots$ that solves HMCP in a limit.

6. Let $\left(x^{*}, \tau^{*}, s^{*}, \kappa^{*}\right)$ be a maximal complementary solution or ray for HMCP. $M C P$ has a solution if and only if $\tau^{*}>0$. In this case, $\left(x^{*} / \tau^{*}, s^{*} / \tau^{*}\right)$ is a complementary solution for MCP.

7. $M C P$ is (strongly) infeasible if and only if $\kappa^{*}>0$. In this case, $\left(x^{*} / \kappa^{*}, s^{*} / \kappa^{*}\right)$ is a certificate (ray) to prove (strong) infeasibility.

We note that if $f$ is an affine function, then there is a strictly complementary solution for HMCP. On the other hand, if $f$ is a monotone function, then the maximal complementary solution $\left(x^{*}, \tau^{*}, s^{*}, \kappa^{*}\right)$ may not be a strictly complementary solution. However, in this case Andersen and Ye [12, proof for Theorem 1] showed that $\tau^{*}+\kappa^{*}>$ 0 . This implies $\left(x^{*}, s^{*}\right)$ must either generate a finite solution for HMCP, or give a certificate that proves the infeasibility of MCP.

2.1. The path following homogeneous algorithm. For simplicity in the following we let $\bar{n}:=n+1, x:=(x ; \tau) \in R_{+}^{\bar{n}}$ and $s:=(s ; \kappa) \in R_{+}^{\bar{n}}$. Let

$$
r^{k}:=s^{k}-F\left(x^{k}\right),
$$


and let $\left(x^{0}, s^{0}\right)=e$ be the starting point. Let $\mathcal{C}(\vartheta)$ denote a continuous trajectory such that

$$
\mathcal{C}(\vartheta):=\left\{\left(x^{k}, s^{k}\right) \mid s^{k}-F\left(x^{k}\right)=\vartheta r^{0}, X^{k} s^{k}=\vartheta e, 0<\vartheta \leq 1\right\} .
$$

Note that such a trajectory always exists (Güler [19]). Moreover, Andersen and Ye [12, Theorem 2] showed that this continuous trajectory is bounded and any limit point is a maximal complementary solution for HMCP. At iteration $k$ with iterate $\left(x^{k}, s^{k}\right)>0$, the algorithm computes the search direction $\left(d_{x}, d_{s}\right)$ by solving the following system of linear equations:

$$
\begin{aligned}
d_{s}-\nabla F\left(x^{k}\right) d_{x} & =-\eta r^{k}, \\
X^{k} d_{s}+S^{k} d_{x} & =\gamma \mu^{k} e-X^{k} s^{k} .
\end{aligned}
$$

Here $\eta$ and $\gamma$ are parameters between 0 and 1 , and $\mu^{k}:=\frac{\left(x^{k}\right)^{T} s^{k}}{\bar{n}}$. For a step size $\alpha>0$, let the new iterate be

$$
\begin{aligned}
x^{+} & :=x^{k}+\alpha d_{x}>0 \text { and } \\
(2.6) s^{+} & \left.:=s^{k}+\alpha d_{s}+\left(F\left(x^{+}\right)-F\left(x^{k}\right)-\alpha \nabla F\left(x^{k}\right) d_{x}\right)\right)=F\left(x^{+}\right)+(1-\alpha \eta) r^{k}>0 .
\end{aligned}
$$

Andersen and Ye [12] showed the following lemmas.

Lemma 2.2. The direction $\left(d_{x}, d_{s}\right)$ satisfies

$$
d_{x}^{T} d_{s}=d_{x}^{T} \nabla F\left(x^{k}\right) d_{x}+\eta(1-\gamma-\eta) \bar{n} \mu^{k} .
$$

Lemma 2.3. Let $r^{+}=s^{+}-F\left(x^{+}\right)$, and consider the new iterate $\left(x^{+}, s^{+}\right)$given by (2.4)-(2.6). Then,

1. $r^{+}=(1-\alpha \eta) r^{k}$.

2. $\left(x^{+}\right)^{T} s^{+}=\left(x^{k}\right)^{T} s^{k}(1-\alpha(1-\gamma))+\alpha^{2} \eta(1-\eta-\gamma) \bar{n} \mu^{k}$.

Note that from Lemma 2.3, for $\eta=1-\gamma$ the infeasibility residual and the complementarity are reduced at the same rate. Based on these lemmas, Andersen and Ye [12] showed that with suitable chosen parameters $\alpha$ and $\beta$, their homogeneous algorithm will generate a sequence $\left\langle x^{k}, s^{k}\right\rangle>0$ such that $\left\langle x^{k}, s^{k}\right\rangle \leq\left\|X^{K} s^{k}-\mu^{k} e\right\| \leq$ $\vartheta \mu^{k}$, where $\vartheta \in(0,1]$ is a constant parameter related to $\nu_{F}(\beta)$. Moreover, they showed that both $\left\|r^{k}\right\|$ and $\left(x^{k}\right)^{T} s^{k}$ converge to zero at a global rate $\gamma=1-\vartheta / \sqrt{\bar{n}}$. As a result, if $f$ satisfies the SLC, then the homogeneous algorithm converges in $\mathcal{O}(\sqrt{n} \log (1 / \epsilon))$ iterations. However, it requires exact search directions and uses short step computations, and thus is not a practical algorithm.

3. A practical potential reduction homogeneous algorithm. We consider the following potential function for the HMCP.

$$
\Phi_{\rho}(x, s):=(\rho / 2) \log \left\{\left(x^{T} s\right)^{2}+\theta\|r\|^{2}\right\}-\sum_{j=1}^{\bar{n}} \log x_{j} s_{j},
$$

where $\theta$ is a constant parameter with positive value and $\rho \geq \bar{n}+\sqrt{\bar{n}}$. A potential function of this form was first introduced by Goldfarb and Mehrotra [17], and was extended to the homogeneous LP model by Mehrotra and Huang [26]. It is similar to 
(1.7) except that the residual vector $r$ is considered in the function. Without loss of generality we choose $\theta=1$ in (3.1).

We need to analyze the worst case decrease in (3.1) at each iteration of our potential reduction IPM. Let

$$
\begin{aligned}
& S^{k}:=\operatorname{diag}\left(s_{1}^{k}, \ldots, s_{\bar{n}}^{k}\right), \quad D:=\left(X^{k} S^{k}\right)^{0.5}, \quad D_{\min }:=\min _{j=1, \ldots, \bar{n}}\left(x_{j}^{k} s_{j}^{k}\right)^{0.5} \\
& p:=\frac{\bar{n}}{\rho} \mu e-D^{2} e=\frac{x^{k} s^{k}}{\rho} e-D^{2} e, \quad \text { and } \\
& \alpha:=\beta \frac{D_{\min }}{\left\|D^{-1} p\right\|}
\end{aligned}
$$

where $\beta \in(0,1)$ is a positive constant that will be determined later. Suppose $\left(d_{x}, d_{s}\right)$ is computed by solving the linear system of equations (2.4) and (2.5) with $\eta=1-\gamma$ and $\gamma=\frac{\bar{n}}{\rho}$. Let

$$
\begin{aligned}
p_{x} & :=\alpha d_{x}, \\
p_{s} & :=\alpha d_{s}+\left(F\left(x^{+}\right)-F\left(x^{k}\right)-\alpha \nabla F\left(x^{k}\right) d_{x}\right), \\
z & :=X^{k}\left(F\left(x^{+}\right)-F\left(x^{k}\right)-\nabla F\left(x^{k}\right) p_{x}\right), \quad \text { and } \\
q & :=z / \alpha,
\end{aligned}
$$

and let $x^{+}:=x^{k}+p_{x}>0$, and $s^{+}:=s^{k}+p_{s}>0$. We have

$$
p=\gamma \mu e-D^{2} e=X^{k} d_{s}+S^{k} d_{x},
$$

and

$$
X^{k} p_{s}+S^{k} p_{x}-\alpha p=z=\alpha q .
$$

In Section 3.1 we show that if $f$ satisfies SLC (and consequently so does $F$ ), then at iteration $k$ the theoretical direction $\left(d_{x}, d_{s}\right)$ with an appropriate step size $\alpha$ results in

$$
\Phi_{\rho}\left(x^{+}, s^{+}\right)-\Phi_{\rho}\left(x^{k}, s^{k}\right) \leq \zeta,
$$

where $\zeta$ is a negative constant. Let $\left(\tilde{x}^{+}, \tilde{s}^{+}\right)$be an iterate computed by using any favored search direction with inexact step sizes in the primal and dual spaces. Starting from an initial solution $\left(x^{0}, s^{0}\right)>0$, our potential reduction IPM is given in Algorithm 1. This algorithm is designed to have the freedom in choosing any favored search direction with inexact and different step sizes in the primal and dual spaces.

In Section 3.2 we show that if $\Phi_{\rho}\left(x^{k}, s^{k}\right)$ is reduced by at least a constant amount at each iteration and $\Phi_{\bar{n}}\left(x^{k}, s^{k}\right)$ (potential function 3.1 with parameter $\rho=\bar{n}$ ) is upper bounded by a constant amount $\iota \bar{n} \log \bar{n}(\iota>0$ is a constant that will be given later), and iterates are maintained while satisfying Assumption 3.1 given below, then Algorithm 1 generates a maximal complementary solution of desired precision in polynomial time.

Assumption $3 . \dot{1}_{\dot{T}}$

(i) $\Omega(1) \leq \frac{\left(x^{0}\right)^{\dot{T}} s^{0}\left|r_{j}^{k}\right|}{\left(x^{k}\right)^{T} s^{k}\left|r_{j}^{0}\right|} \leq \mathcal{O}(1), j=1, \ldots, \bar{n}$

(ii) $\Omega(1) \leq \frac{\left(\tilde{x}^{+}\right)^{T} \tilde{s}^{+}}{\left(x^{+}\right)^{T} s^{+}} \leq \mathcal{O}(1)$.

Assumption 3.1 (i) requires that the overall decrease in the infeasibility to complementarity ratio is maintained in Algorithm 1. Assumption 3.1 (ii) requires that the complementarity at the iterate $\left(\tilde{x}^{+}, \tilde{s}^{+}\right)$generated using a favored direction and 


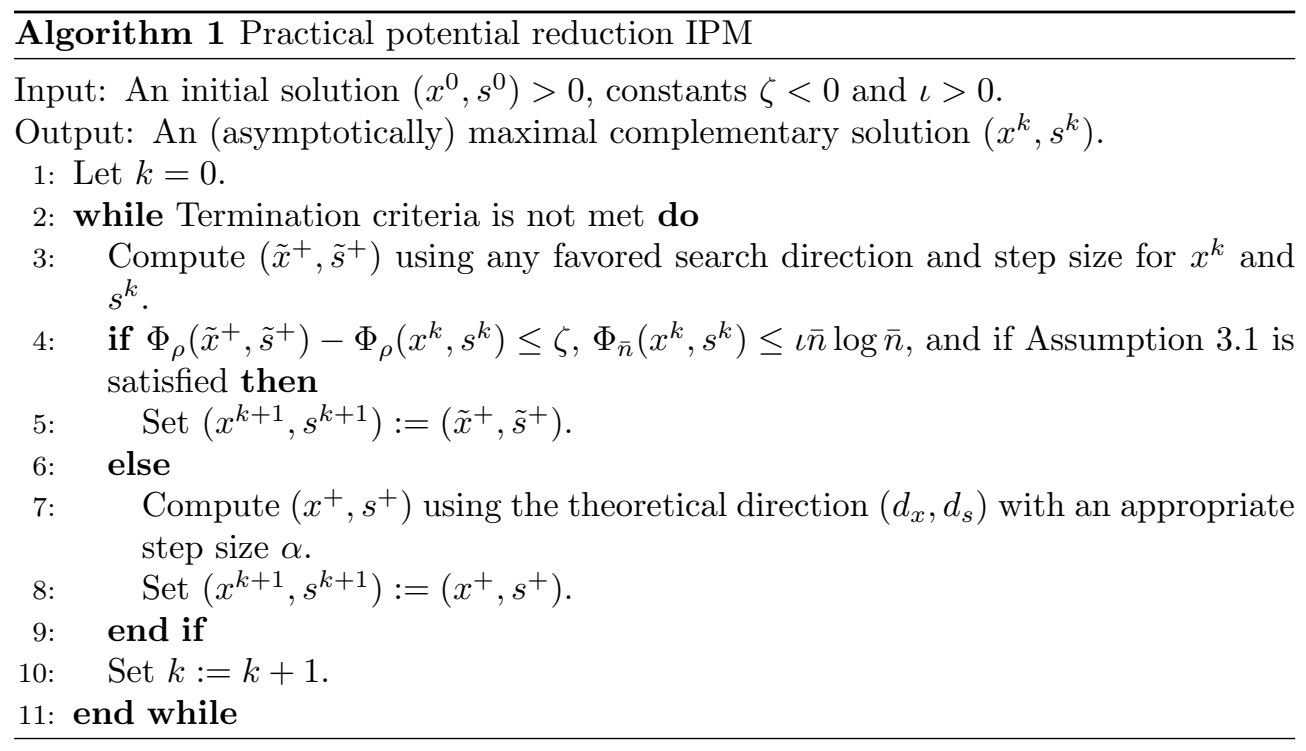

the theoretical iterate $\left(x^{+}, s^{+}\right)$are of the same order. Note that if $\eta=1-\gamma$, then from Lemma 2.3 we have $\left(x^{+}\right)^{T} s^{+}=(1-\alpha \eta)\left(x^{k}\right)^{T} s^{k}$. As a result if only theoretical direction is chosen in the algorithm, Assumption 3.1 is satisfied with constant equal to 1 , and the complementarity is decreased monotonically. Convergence properties for an algorithm that has additional flexibility of choosing more aggressive directions and step size are still ensured under Assumption 3.1.

3.1. Potential function reduction using the theoretical direction. The following analysis follows some of the steps in Kojima et al. [22] and Potra and Ye [31, Sections 2 and 3]. We drop the index $k$ for an iteration to simplify the presentation.

Proposition 3.2 (Kojuma et al. [22, Lemma 2.3]). Let $u, v$, and $w \in R^{\bar{n}}$ satisfy $u+v=w$ and $u^{T} v \geq 0$. Then

$$
\|u\| \leq\|w\|,\|v\| \leq\|w\|, \text { and } u^{T} v \leq \frac{1}{2}\|w\|^{2} .
$$

Lemma 3.3. Let $p_{x}$ be defined as in (3.3). Then, $\left\|X^{-1} p_{x}\right\|_{\infty} \leq \beta$.

Proof. We use the technique in Kojima et al. [22] for the proof. We first rewrite equation (3.4) as

$$
\frac{D^{-1} X d_{s}}{\left\|D^{-1} p\right\|}+\frac{D^{-1} S d_{x}}{\left\|D^{-1} p\right\|}=\frac{D^{-1} p}{\left\|D^{-1} p\right\|} .
$$

From Lemma 2.2, we have

$$
\frac{\left(D^{-1} X d_{s}\right)^{T} D^{-1} S d_{x}}{\left\|D^{-1} p\right\|\left\|D^{-1} p\right\|}=\frac{d_{x}^{T} d_{s}}{\left\|D^{-1} p\right\|^{2}} \geq 0 .
$$

Now using Proposition 3.2 in (3.6) and (3.7), we have

$$
\frac{\left\|D^{-1} S d_{x}\right\|}{\left\|D^{-1} p\right\|} \leq \frac{\left\|D^{-1} p\right\|}{\left\|D^{-1} p\right\|}=1 .
$$


Using (3.3) and (3.8), we deduce the following relation

$$
\frac{\left\|X^{-1} d_{x}\right\|}{\left\|D^{-1} p\right\|}=\frac{\left\|D^{-2} S d_{x}\right\|}{\left\|D^{-1} p\right\|} \leq D_{\min }^{-1}
$$

Finally, using (3.2) and (3.9), we have

$$
\left\|X^{-1} p_{x}\right\|_{\infty}=\alpha\left\|X^{-1} d_{x}\right\|_{\infty} \leq \beta D_{\min } \frac{\left\|X^{-1} d_{x}\right\|}{\left\|D^{-1} p\right\|} \leq \beta .
$$

Potra and Ye [31] showed the following lemma.

Lemma 3.4 (Potra and Ye [31, Lemma 3.2]). If $d_{x}$ and $d_{s}$ are the solution of the linear system (3.5), and if condition (1.6) and Lemma (3.3) are satisfied, then

$$
\|z\| \leq \xi \beta^{2} D_{\min }^{2}
$$

where $\xi=0.25 \nu_{F}(\beta)$ and $z$ is defined in (3.3).

From the above lemma and (3.2) we have

$$
\left\|D^{-1} q\right\| \leq\|q\| D_{\min }^{-1}=\frac{\|z\|}{\alpha} D_{\min }^{-1} \leq \frac{\xi \beta^{2}}{\alpha} D_{\min }=\xi \beta\left\|D^{-1} p\right\| .
$$

Kojima et al. showed the following lemma.

Lemma 3.5 (Kojima et al. [22, Lemma 2.5]). Suppose the search direction $\left(d_{x}, d_{s}\right)$ is computed by solving the linear system of equations (2.4) and (2.5) with $\eta=1-\gamma$ and $\gamma=\frac{\bar{n}}{\rho}(\rho \geq \bar{n}+\sqrt{\bar{n}})$, then

$$
\frac{\rho}{x^{T} s}\left\|D^{-1} p\right\|=\left\|D^{-1} e-\frac{\rho}{x^{T} s} D e\right\| \geq \frac{\sqrt{3}}{2} D_{\min }^{-1} .
$$

In the following we define two real valued functions $g_{1}$ and $g_{2}$, and establish results that upper bound them. These two functions and the related upper bounds will be used later in our analysis. Let

$$
\begin{aligned}
& g_{1}:=-\alpha \rho \eta+e^{T}\left(X^{-1} p_{x}+S^{-1} p_{s}\right) \text { and } \\
& g_{2}:=\frac{\left\|X^{-1} p_{x}\right\|^{2}+\left\|S^{-1} p_{s}\right\|^{2}}{2(1-\beta)} .
\end{aligned}
$$

Lemma 3.6. Let $g_{1}$ be defined as in (3.11). With a suitably chosen parameter $\beta \in(0,1)$, suppose the search direction $\left(d_{x}, d_{s}\right)$ is computed by solving the linear system of equations (2.4) and (2.5) with $\eta=1-\gamma$ and $\gamma=\frac{\bar{n}}{\rho}(\rho \geq \bar{n}+\sqrt{\bar{n}})$. Let the step size $\alpha$ be defined as in (3.2). If condition (1.6) is satisfied, then

$$
g_{1} \leq-\frac{\sqrt{3}}{2}(1-\xi \beta) \beta+\xi \beta^{2} \frac{\rho}{\sqrt{\bar{n}}} .
$$

Proof. From (2.5) and $\gamma=1-\eta$, we have

$$
-\alpha \rho \eta=\alpha \rho \frac{x^{T} d_{s}+s^{T} d_{x}}{\bar{n} \mu} .
$$


An upper bound of $g_{1}$ is obtained in the following set of inequalities.

$$
\begin{aligned}
& g_{1}=\alpha \rho\left(\frac{x^{T} d_{s}+s^{T} d_{x}}{x^{T} s}\right)-e^{T}\left(X^{-1} p_{x}+S^{-1} p_{s}\right) \\
& =\alpha \frac{\rho}{x^{T} s} e^{T}\left(X d_{s}+S d_{x}\right)-e^{T} D^{-2}\left(X p_{s}+S p_{x}\right) \\
& =\alpha \frac{\rho}{x^{T} s} e^{T}\left(p-\frac{x^{T} s}{\rho} D^{-2}(p+q)\right) \quad(\text { using }(3.4) \text { and }(3.5)) \\
& =\alpha \frac{\rho}{x^{T} s} e^{T}\left(\left(D-\frac{x^{T} s}{\rho} D^{-1}\right) D^{-1} p-\frac{x^{T} s}{\rho} D^{-2} q\right) \\
& =-\alpha \frac{\rho}{x^{T} s}\left(p^{T} D^{-1} D^{-1} p\right)-\alpha \frac{\rho}{x^{T} s}\left(D^{-1} p+D e\right)^{T} D^{-1} q \quad \text { (using (3.2)) } \\
& =-\alpha \frac{\rho}{x^{T} s}\left(p^{T} D^{-1} D^{-1}(p+q)\right)-\alpha \frac{\rho}{x^{T} s} e^{T} q \\
& =-\alpha \frac{\rho}{x^{T} s}\left(\left\|D^{-1} p\right\|^{2}+p^{T} D^{-1} D^{-1} q\right)-\frac{\rho}{x^{T} s} e^{T} z \quad \text { (using (3.3)) } \\
& \leq-\alpha \frac{\rho}{x^{T} s}\left(\left\|D^{-1} p\right\|^{2}-\left\|D^{-1} p\right\|\left\|D^{-1} q\right\|\right)+\frac{\rho}{x^{T} s}\|z\|_{1} \\
& \leq-\alpha \frac{\rho}{x^{T} s}\left(\left\|D^{-1} p\right\|^{2}-\xi \beta\left\|D^{-1} p\right\|^{2}\right)+\frac{\rho}{x^{T} s} \sqrt{\bar{n}}\|z\| \quad \text { (using (3.10)) } \\
& \leq-\alpha \frac{\rho}{x^{T} s}(1-\xi \beta)\left\|D^{-1} p\right\|^{2}+\frac{\rho}{x^{T} s} \sqrt{\bar{n}} \xi \beta^{2} D_{\text {min }}^{2} \quad \text { (using Lemma 3.4) } \\
& \leq-\frac{\sqrt{3}}{2}(1-\xi \beta) \alpha D_{\min }^{-1}\left\|D^{-1} p\right\|+\frac{\rho}{\sqrt{\bar{n}}} \xi \beta^{2} \quad \text { (using Lemma 3.5) } \\
& =-\frac{\sqrt{3}}{2}(1-\xi \beta) \beta+\frac{\rho}{\sqrt{\bar{n}}} \xi \beta^{2} \text {. (using (3.2)) }
\end{aligned}
$$

LEMma 3.7. Let $_{2}$ be defined as in (3.12). Under the hypothesis of Lemma 3.7, we have

$$
g_{2} \leq \frac{\beta^{2}(1+\xi \beta)^{2}}{2(1-\beta)}
$$

Proof. The following proof is based on techniques in Potra and Ye [31]. We have

$$
\begin{aligned}
2(1-\beta) g_{2} & =\left\|X^{-1} p_{x}\right\|^{2}+\left\|S^{-1} p_{s}\right\|^{2} \\
& =\left\|D^{-2} X p_{s}\right\|^{2}+\left\|D^{-2} S p_{x}\right\|^{2} \\
& \leq D_{\min }^{-2}\left(\left\|D^{-1} X p_{s}\right\|^{2}+\left\|D^{-1} S p_{x}\right\|^{2}\right) \\
& =D_{\min }^{-2}\left(\left\|D^{-1}\left(X p_{s}+S p_{x}\right)\right\|^{2}-2 p_{x}^{T} p_{s}\right) \\
& =D_{\min }^{-2}\left(\alpha^{2}\left\|D^{-1}(p+q)\right\|^{2}-2 p_{x}^{T} p_{s}\right) \quad(\text { using }(3.5)) \\
& \leq D_{\min }^{-2}\left(\alpha^{2}\left(\left\|D^{-1} p\right\|+\left\|D^{-1} q\right\|\right)^{2}-2 p_{x}^{T} p_{s}\right) \\
& \leq D_{\min }^{-2}\left(\alpha^{2}(1+\xi \beta)^{2} \mid D^{-1} p \|^{2}-2 p_{x}^{T} p_{s}\right) \quad(\text { using }(3.10)) \\
& =\left(\alpha D_{\min }^{-1}\left\|D^{-1} p\right\|\right)^{2}(1+\xi \beta)^{2}-2 D_{\min }^{-2} p_{x}^{T} p_{s} \\
& =\beta^{2}(1+\xi \beta)^{2}-2 D_{\min }^{-2} p_{x}^{T} p_{s} . \quad \quad \quad(\operatorname{using}(3.2))
\end{aligned}
$$

The upper bound on $p_{x}^{T} p_{s}$ is obtained as follows.

$(3.15) p_{x}^{T} p_{s}=p_{x}^{T}\left(\alpha d_{s}+\left(F\left(x^{+}\right)-F(x)-\nabla F(x) p_{x}\right)\right) \quad($ using $(3.3))$ 


$$
\begin{aligned}
& =\left(x^{+}-x\right)^{T}\left(F\left(x^{+}\right)-F(x)\right)+\alpha^{2}\left(d_{x}^{T} d_{s}-d_{x}^{T} \nabla F(x) d_{x}\right) \quad \text { (using (3.3)) } \\
& =\left(x^{+}-x\right)^{T}\left(F\left(x^{+}\right)-F(x)\right)+\alpha^{2} \eta(1-\eta-\gamma) \bar{n} \mu \quad \text { (using Lemma 2.2) } \\
& \geq 0 \quad(\text { from the monotonicity of } F) .
\end{aligned}
$$

The result follows from using (3.14) and (3.15).

The following proposition is frequently used in the IPM analysis.

Proposition 3.8 (Karmarker [21]).

1. If $1-\delta>0$, then $\log \{1-\delta\} \leq-\delta$.

2. If $\delta \in R^{\bar{n}}$ satisfies $\|\delta\|_{\infty} \leq \beta<1$, then $\sum_{j=1}^{\bar{n}} \log \left\{1-\delta_{j}\right\} \geq-e^{T} \delta-\frac{\|\delta\|^{2}}{2(1-\beta)}$.

Now we are ready to show a bound on the difference between the potential function values $\Phi_{\rho}\left(x^{+}, s^{+}\right)$and $\Phi_{\rho}(x, s)$. The next theorem bounds the improvement in the potential function from taking the theoretical direction with a controllable step size.

TheOREM 3.9. Let $\xi=0.25 \nu_{F}(\beta) \geq 0.25$. Suppose the search direction $\left(d_{x}, d_{s}\right)$ is computed by solving the linear system of equations (2.4) and (2.5) with $\eta=1-\gamma$ and $\gamma=\frac{\bar{n}}{\rho}(\rho \geq \bar{n}+\sqrt{\bar{n}})$. Let the step size $\alpha$ be defined as in (3.2). If condition (1.6) is satisfied, and $\beta \in(0,0.303)$ is chosen such that

$$
\begin{aligned}
\xi \beta & \leq \frac{\sqrt{3}}{4(\sqrt{3}+2 \rho / \sqrt{\bar{n}})}, \quad \text { and } \\
\beta \sqrt{\xi}(1+\xi \beta) & \leq \frac{\sqrt{3}}{2(\sqrt{3}+2 \rho / \sqrt{\bar{n}})^{1 / 2}} .
\end{aligned}
$$

Then,$\left(x^{+}, s^{+}\right)$is always strictly positive. Moreover,

$$
\Phi_{\rho}\left(x^{+}, s^{+}\right)-\Phi_{\rho}(x, s) \leq-0.278 \frac{\beta}{1-\beta} .
$$

Proof. We first show that $\left(x^{+}, s^{+}\right)$is strictly positive. From Lemma 3.7 and (3.17), we have

$$
\max \left\{\left\|X^{-1} p_{x}\right\|_{\infty},\left\|S^{-1} p_{s}\right\|_{\infty}\right\} \leq \beta(1+\xi \beta) \leq \frac{\sqrt{3}}{2(\xi(\sqrt{3}+2 \rho / \sqrt{\bar{n}}))^{1 / 2}}<1 .
$$

Hence, $\left(p_{x}, p_{s}\right)$ is a valid direction. We note that from Lemma 3.3 , we have

$$
\left\|X^{-1} p_{x}\right\|_{\infty} \leq \beta \leq \frac{\sqrt{3}}{4 \xi(\sqrt{3}+2 \rho / \sqrt{\bar{n}})} \leq \frac{\sqrt{3}}{2(\xi(\sqrt{3}+2 \rho / \sqrt{\bar{n}}))^{1 / 2}} .
$$

Therefore, the upper bound from Lemma 3.3 is still valid (and tighter). Moreover, $\beta<0.303$ implies that the condition in Theorem 2.1 [4] is satisfied. Now,

$$
\begin{aligned}
& \Phi_{\rho}\left(x^{+}, s^{+}\right)-\Phi_{\rho}(x, s) \\
= & (\rho / 2) \log \left\{(1-\alpha \eta)^{2}\left((\bar{n} \mu)^{2}+\|r\|^{2}\right)\right\}-\sum_{j=1}^{\bar{n}} \log \left\{\left(x_{j}+\left(p_{x}\right)_{j}\right)\left(s_{j}+\left(p_{s}\right)_{j}\right)\right\} \\
- & (\rho / 2) \log \left\{(\bar{n} \mu)^{2}+\|r\|^{2}\right\}+\sum_{j=1}^{\bar{n}} \log \left\{x_{j} s_{j}\right\} \text { (using Lemma 2.3) }
\end{aligned}
$$




$$
\begin{aligned}
& =\rho \log \{1-\alpha \eta\}-\sum_{j=1}^{\bar{n}} \log \left\{1+\frac{\left(p_{x}\right)_{j}}{x_{j}}\right\}\left\{1+\frac{\left(p_{s}\right)_{j}}{s_{j}}\right\} \\
& \leq-\alpha \rho \eta-e^{T}\left(X^{-1} p_{x}+S^{-1} p_{s}\right)-\frac{\left\|X^{-1} p_{x}\right\|^{2}+\left\|S^{-1} p_{s}\right\|^{2}}{2(1-\beta)} \text { (using Proposition 3.8) } \\
& =g_{1}+g_{2} .
\end{aligned}
$$

From Lemmas 3.6 and 3.7, we write

$$
\begin{aligned}
\Phi_{\rho}\left(x^{+}, s^{+}\right)-\Phi_{\rho}(x, s) & \leq-\frac{\sqrt{3}}{2}(1-\xi \beta) \beta+\xi \beta^{2} \rho / \sqrt{\bar{n}}+\frac{\beta^{2}(1+\xi \beta)^{2}}{2(1-\beta)} \\
& =\frac{\beta^{2}(1+\xi \beta)^{2}}{2(1-\beta)}+\beta\left(-\frac{\sqrt{3}}{2}+\xi \beta\left(\frac{\sqrt{3}}{2}+\frac{\rho}{\sqrt{\bar{n}}}\right)\right) \\
& =\frac{\beta^{2}(1+\xi \beta)^{2}}{2(1-\beta)}-\frac{\sqrt{3}}{2} \beta\left(1-\xi \beta\left(\frac{\sqrt{3}+2(\rho / \sqrt{\bar{n}})}{\sqrt{3}}\right)\right)=: \zeta .
\end{aligned}
$$

It follows that

$$
-2 \frac{1-\beta}{\beta} \zeta=-\beta(1+\xi \beta)^{2}+\sqrt{3}(1-\beta)\left(1-\xi \beta\left(\frac{\sqrt{3}+2(\rho / \sqrt{\bar{n}})}{\sqrt{3}}\right)\right) .
$$

Numerically, it is easy to verify that

$$
\zeta \leq-0.278 \frac{\beta}{1-\beta} .
$$

Since $\nu_{F}(\beta):(0,1) \mapsto(1, \infty)$ is a monotone increasing function depending on $\beta \in(0,0.303)$, we can always choose a sufficiently small $\beta$ that satisfies (3.16) and (3.17). As a result, a reduction in the potential function $\left(\zeta<-0.278 \frac{\beta}{1-\beta}\right)$ is guaranteed. However, choosing $\beta$ is dependent on $\nu_{F}(\beta)$, finding a maximal $\beta$ that satisfies (3.16) and (3.17) requires the knowledge of the scaled Lipschitz constant $\nu_{F}(\beta)$, which may not be available in practice for an arbitrary convex function. Problems satisfying the scaled Lipschitz condition are discussed in [27, 30, 41]. Nevertheless, the analysis suggests that for an appropriate step size, the potential function value will reduce as long as the scaled Lipschitz constant is bounded. Consequently, the potential function can be used as a guide to solve monotone complementarity and convex optimization problems satisfying this property. If the condition is not satisfied, then lack of sufficient reduction in the potential function indicates that the algorithm proposed here is not appropriate for the problem being solved. Finally, we note that Theorem 3.9 is similar to the result by Potra and Ye [31], but with a difference that it allows us to choose a larger $\rho$, when $\beta$ is sufficiently small.

Remark. Note that by performing simple block elimination on the system of linear equations (2.4) and (2.5), we obtain the following alternative formulation:

$$
X\left(\alpha \nabla F(x) d_{x}-\alpha \eta r\right)+\alpha S d_{x}-\alpha p=0 .
$$

Hence, $d_{x}$ is a direction obtained by applying a single Newton step to the equation

$$
z(h):=X(F(x+\alpha h)-F(x)-\alpha \eta r)+\alpha S h-\alpha p=0,
$$


with starting point $h=0$. If $F$ is linear (for example, LP formulated as a HMCP model), then the linear system (3.19) can be solved exactly. In this case Theorem 3.9 with $\xi=0$ reduces to the result of Mehrotra and Huang [26].

Suppose $F$ is nonlinear and does not satisfy SLC. Given positive constants $\xi$ and $\beta$ that satisfy conditions (3.16) and (3.17). If one can compute an approximate solution $\delta_{x}$ such that

$$
\left\|z\left(\delta_{x}\right)\right\|=\left\|X\left(F\left(x+\alpha \delta_{x}\right)-F(x)-\alpha \eta r\right)+\alpha S \delta_{x}-\alpha p\right\| \leq \xi \beta^{2} \min _{j=1, \ldots, \bar{n}}\left(x_{j} s_{j}\right),
$$

then the result of Theorem 3.9 is still satisfied. However, finding a direction $\delta_{x}$ may require many Newton steps, and an explicit bound on the number of Newton steps is unknown.

3.2. Convergence of the practical potential reduction homogeneous algorithm. The following theorem shows that if potential function (3.1) can be reduced by at least a constant amount at each iteration, then the Algorithm 1 generates an $\epsilon$-complementary solution in polynomial time. Our analysis follows some of the steps in Kojima et al. [22] and Porta and Ye [29].

TheOREm 3.10. Let $\epsilon>0$. Starting from a solution $\left(x^{0}, s^{0}\right)>0$, if the value of potential function (3.1) can be reduced by at least a constant amount and if Assumption 3.1 is satisfied at each iteration of Algorithm 1, then after at most $\mathcal{O}\left(\Phi_{\rho}\left(x^{0}, s^{0}\right)-\right.$ $\bar{n} \log \bar{n}+(\rho-\bar{n})|\log \epsilon|)$ iterations, we will have a solution such that

$$
x^{T} s \leq \epsilon \text { and } \frac{\left|r_{j}\right|}{\left|r_{j}^{0}\right|} \leq \mathcal{O}(1) \frac{x^{T} s}{\left(x^{0}\right)^{T} s^{0}}, j=1, \ldots, \bar{n} .
$$

Proof. Using Assumption 3.1, we have

$$
(\rho / 2) \log \left\{\left(x^{T} s\right)^{2}\left(1+\left(\Omega(1) \frac{\left\|r^{0}\right\|}{\left(x^{0}\right)^{T} s^{0}}\right)^{2}\right)\right\}-\sum_{j=1}^{\bar{n}} \log x_{j} s_{j} \leq \Phi_{\rho}(x, s),
$$

which implies

$$
\begin{aligned}
& (\rho-\bar{n}) \log x^{T} s-\sum_{j=1}^{\bar{n}} \log \left\{\frac{\bar{n} x_{j} s_{j}}{x^{T} s}\right\}+(\rho / 2) \log \left\{1+\left(\Omega(1) \frac{\left\|r^{0}\right\|}{\left(x^{0}\right)^{T} s^{0}}\right)^{2}\right\} \\
& \leq \Phi_{\rho}(x, s)-\bar{n} \log \bar{n} .
\end{aligned}
$$

Since the second and the third terms of (3.22) are always nonnegative, it follows that

$$
(\rho-\bar{n}) \log x^{T} s \leq \Phi_{\rho}(x, s)-\bar{n} \log \bar{n} .
$$

Therefore, $\Phi_{\rho}(x, s) \leq \bar{n} \log \bar{n}-(\rho-\bar{n})|\log \epsilon|$, then we have

$$
x^{T} s \leq \epsilon .
$$

Moreover, Assumption 3.1 implies that

$$
\frac{\left|r_{j}\right|}{\left|r_{j}^{0}\right|} \leq \mathcal{O}(1) \frac{x^{T} s}{\left(x^{0}\right)^{T} s^{0}}, j=1, \ldots, \bar{n} .
$$


We now show that any limit point of a sequence $\left\langle x^{k}, s^{k}\right\rangle$ generated by the potential reduction algorithm of Section 3.2 is a maximal complementary solution. Our analysis here follows some of the steps in Güler and Ye [20, Sections 2 and 4] and Potra and Ye $[31$, Section 4].

Lemma 3.11. Given an initial positive point $\left(x^{0}, s^{0}\right)$. Recall that $\left(\tilde{x}^{+}, \tilde{s}^{+}\right)$is a tentative point computed using any favored search direction using an inexact and different step size in the primal and dual spaces, and it satisfies Assumption 3.1; whereas $\left(x^{+}, s^{+}\right)$is computed using the theoretical direction $\left(d_{x}, d_{s}\right)$ and a fixed step size $\alpha$. Let

$$
\begin{aligned}
C_{1} & :=\frac{\rho-\bar{n}}{2} \log \left\{\frac{\left(\left(x^{0}\right)^{T} s^{0}\right)^{2}+\mathcal{O}(1)^{2}\left\|r^{0}\right\|^{2}}{\left(\left(x^{0}\right)^{T} s^{0}\right)^{2}+\Omega(1)^{2}\left\|r^{0}\right\|^{2}}\right\} \geq 0, \quad \text { and } \\
C_{2} & :=C_{1}+\max \{0,-(\rho-\bar{n}) \log \Omega(1)\} \geq 0,
\end{aligned}
$$

then,

$$
\begin{aligned}
& \Phi_{\bar{n}}\left(x^{+}, s^{+}\right)-\Phi_{\bar{n}}\left(x^{k}, s^{k}\right) \leq \zeta-(\rho-\bar{n}) \log \left\{1-D_{\min }^{2} \frac{\sqrt{\bar{n}}}{\left(x^{k}\right)^{T} s^{k}}\right\}, \quad \text { and } \\
& \Phi_{\bar{n}}\left(\tilde{x}^{+}, \tilde{s}^{+}\right)-\Phi_{\bar{n}}\left(x^{k}, s^{k}\right) \leq \zeta-(\rho-\bar{n}) \log \left\{1-D_{\min }^{2} \frac{\sqrt{\bar{n}}}{\left(x^{k}\right)^{T} s^{k}}\right\}+C_{2},
\end{aligned}
$$

where $\Phi_{\bar{n}}\left(x^{k}, s^{k}\right)$ is defined as in (3.1) with $\rho=\bar{n}$.

Proof. To simplify the presentation we drop the index $k$. Using Assumption 3.1, we have

$$
\begin{aligned}
& \left\|\tilde{r}^{+}\right\|^{2} \geq\left(\Omega(1)\left\|r^{0}\right\| \frac{\left(\tilde{x}^{+}\right)^{T} \tilde{s}^{+}}{\left(x^{0}\right)^{T} s^{0}}\right)^{2},\|r\|^{2} \leq\left(\mathcal{O}(1)\left\|r^{0}\right\| \frac{x^{T} s}{\left(x^{0}\right)^{T} s^{0}}\right)^{2}, \text { and } \\
& \left(\tilde{x}^{+}\right)^{T} \tilde{s}^{+} \geq \Omega(1)\left(x^{+}\right)^{T} s^{+} .
\end{aligned}
$$

First we bound the difference between the values of $\Phi_{\bar{n}}\left(\tilde{x}^{+}, \tilde{s}^{+}\right)$and $\Phi_{\bar{n}}(x, s)$. Let $\tilde{r}^{+}=\tilde{s}^{+}-F\left(\tilde{x}^{+}\right)$.

$$
\begin{aligned}
& \Phi_{\bar{n}}\left(\tilde{x}^{+}, \tilde{s}^{+}\right)-\Phi_{\bar{n}}(x, s) \\
= & \Phi_{\rho}\left(\tilde{x}^{+}, \tilde{s}^{+}\right)-\Phi_{\rho}(x, s)-\Phi_{\rho}\left(\tilde{x}^{+}, \tilde{s}^{+}\right)+\Phi_{\rho}(x, s)+\Phi_{\bar{n}}\left(\tilde{x}^{+}, \tilde{s}^{+}\right)-\Phi_{\bar{n}}(x, s) \\
\leq & \zeta-\frac{\rho-\bar{n}}{2} \log \left\{\left(\left(\tilde{x}^{+}\right)^{T} \tilde{s}^{+}\right)^{2}+\left\|\tilde{r}^{+}\right\|^{2}\right\} \\
& \quad+\frac{\rho-\bar{n}}{2} \log \left\{\left(x^{T} s\right)^{2}+\|r\|^{2}\right\} \text { (using Theorem 3.9) } \\
\leq & \zeta-\frac{\rho-\bar{n}}{2} \log \left\{\left(\left(\tilde{x}^{+}\right)^{T} \tilde{s}^{+}\right)^{2}+\left(\Omega(1)\left\|r^{0}\right\| \frac{\left(\tilde{x}^{+}\right)^{T} \tilde{s}^{+}}{\left(x^{0}\right)^{T} s^{0}}\right)^{2}\right\} \\
& +\frac{\rho-\bar{n}}{2} \log \left\{\left(x^{T} s\right)^{2}+\left(\mathcal{O}(1)\left\|r^{0}\right\| \frac{x^{T} s}{\left(x^{0}\right)^{T} s^{0}}\right)^{2}\right\}(\text { using (3.25)) } \\
=\zeta- & (\rho-\bar{n}) \log \left\{\frac{\left(\tilde{x}^{+}\right)^{T} \tilde{s}^{+}}{x^{T} s}\right\}+C_{1} \\
\leq & \zeta-(\rho-\bar{n}) \log \left\{\frac{\left(x^{+}\right)^{T} s^{+}}{x^{T} s}\right\}-(\rho-\bar{n}) \log \Omega(1)+C_{1} \text { (using (3.26)) }
\end{aligned}
$$




$$
\begin{aligned}
& \leq \zeta-(\rho-\bar{n}) \log \left\{\frac{\left(x^{+}\right)^{T} s^{+}}{x^{T} s}\right\}+C_{2} \\
& \leq \zeta-(\rho-\bar{n}) \log \{1-\alpha \eta\}+C_{2} \text { (using Lemma 2.3) } \\
& =\zeta-(\rho-\bar{n}) \log \left\{1+\alpha \frac{x^{T} d_{s}+s^{T} d_{s}}{x^{T} s}\right\}+C_{2} \text { (using (3.13)) } \\
& =\zeta-(\rho-\bar{n}) \log \left\{1+\alpha \frac{p^{T} e}{x^{T} s}\right\}+C_{2}(\text { using (3.4)). }
\end{aligned}
$$

Now,

$$
\begin{aligned}
\alpha \frac{p^{T} e}{x^{T} s} & =\frac{\alpha\left(\left(x^{T} s / \rho\right) e^{T} e-e^{T} D^{2} e\right)}{x^{T} s}(\operatorname{using}(3.2)) \\
& =\alpha\left(\frac{\bar{n}}{\rho}-1\right) \\
& =-\beta \frac{D_{\min }}{\left\|D^{-1} p\right\|}\left(\frac{\rho-\bar{n}}{\rho}\right)(\operatorname{using}(3.2)) \\
& \geq-\beta D_{\min }^{2} \frac{2(\rho-\bar{n})}{\sqrt{3} x^{T} s}(\text { using Lemma } 3.5) \\
& \geq-D_{\min }^{2} \frac{\sqrt{\bar{n}}(\rho-\bar{n})}{2 x^{T} s \xi(\sqrt{3 \bar{n}}+2 \rho)}(\text { using }(3.16)) \\
& \geq-D_{\min }^{2} \frac{2 \rho \sqrt{\bar{n}}}{x^{T} s(\sqrt{3 \bar{n}}+2 \rho)}\left(\text { because } \xi=0.25 \nu_{F} \beta \geq 0.25\right) \\
& \geq-D_{\min }^{2} \frac{\sqrt{\bar{n}}}{x^{T} s} .
\end{aligned}
$$

Therefore,

$$
\Phi_{\bar{n}}\left(\tilde{x}^{+}, \tilde{s}^{+}\right)-\Phi_{\bar{n}}(x, s) \leq \zeta-(\rho-\bar{n}) \log \left\{1-D_{\min }^{2} \frac{\sqrt{\bar{n}}}{x^{T} s}\right\}+C_{2} .
$$

The difference between the values of $\Phi_{\bar{n}}\left(x^{+}, s^{+}\right)$and $\Phi_{\bar{n}}(x, s)$ can be constructed in a similar way.

Lemma 3.12. Given an initial positive point $\left(x^{0}, s^{0}\right)$. Suppose a sequence $\left\langle x^{k}, s^{k}\right\rangle$ is generated by using Algorithm 1. There is a constant $M_{1}$ independent of $k$ such that

$$
\Phi_{\bar{n}}\left(x^{k}, s^{k}\right) \leq M_{1} \text { for all } k \text {. }
$$

Proof. Let $C_{3}:=(\bar{n} / 2) \log \left\{1+\left(\mathcal{O}(1) \frac{\left\|r^{0}\right\|}{\left(x^{0}\right)^{T} s^{0}}\right)^{2}\right\}>0$. Using (3.25), we have $\Phi_{\bar{n}}(x, s)=(\bar{n} / 2) \log \left\{\left(x^{T} s\right)^{2}+\|r\|^{2}\right\}-\sum_{j=1}^{\bar{n}} \log x_{j} s_{j}$ $\leq(\bar{n} / 2) \log \left\{\left(x^{T} s\right)^{2}+\left(\mathcal{O}(1)\left\|r^{0}\right\| \frac{x^{T} s}{\left(x^{0}\right)^{T} s^{0}}\right)^{2}\right\}-\sum_{j=1}^{\bar{n}} \log x_{j} s_{j}($ using (3.25)) $(3.27)=\bar{n} \log x^{T} s-\sum_{j=1}^{\bar{n}} \log x_{j} s_{j}+C_{3}$. 
In the following we analyze the behavior of $\bar{n} \log x^{T} s-\sum_{j=1}^{\bar{n}} \log x_{j} s_{j}$. Let $\iota>0$ be sufficiently large so that

$$
\zeta-(\rho-\bar{n}) \log \left\{1-\frac{1}{\bar{n}^{\iota-0.5}}\right\}<0 .
$$

Now consider the case where $\Phi_{\bar{n}}(x, s) \geq \bar{n} \log x^{T} s-\sum_{j=1}^{\bar{n}} \log x_{j} s_{j} \geq \iota \bar{n} \log \bar{n}$. In this case we have,

$$
\begin{aligned}
& \bar{n} \log x^{T} s+\bar{n} \log D_{\min }^{-2} \geq \iota \bar{n} \log \bar{n}, \\
\Rightarrow & \log \left\{x^{T} s D_{\min }^{-2}\right\} \geq \iota \log \bar{n}, \\
\Rightarrow & \Phi_{\bar{n}}\left(x^{+}, s^{+}\right)-\Phi_{\bar{n}}(x, s)=\zeta-(\rho-\bar{n}) \log \left\{1-D_{\min }^{2} \frac{\sqrt{\bar{n}}}{x^{T} s}\right\} \quad \text { (using (3.23)) } \\
& \leq \zeta-(\rho-\bar{n}) \log \left\{1-\frac{1}{\bar{n}^{\iota-0.5}}\right\}<0 .
\end{aligned}
$$

The above relations suggests that using the theoretical direction and a fixed step size can decrease the value of $\Phi_{\bar{n}}(x, s)$ if this value is larger than $\iota \bar{n} \log \bar{n}$. Next we consider the case where $\Phi_{\bar{n}}(x, s) \leq \iota \bar{n} \log \bar{n}$. We show that the value of $\Phi_{\bar{n}}(x, s)$ in this case is upper bounded by a constant amount independent of $k$, and the iterate is computed using any favored search direction with inexact and different step sizes in the primal and dual spaces.

Using (3.24) and (3.27), we have

$$
\begin{aligned}
\Phi_{\bar{n}}\left(\tilde{x}^{+}, \tilde{s}^{+}\right) & \leq \Phi_{\bar{n}}(x, s) \leq \iota \bar{n} \log \bar{n}+C_{3} \\
& \leq \zeta-(\rho-\bar{n}) \log \left\{1-D_{\min }^{2} \frac{\sqrt{\bar{n}}}{x^{T} s}\right\}+\iota \bar{n} \log \bar{n}+C_{2}+C_{3} \\
& \leq \zeta-(\rho-\bar{n}) \log \left\{1-\frac{1}{\sqrt{\bar{n}}}\right\}+\iota \bar{n} \log \bar{n}+C_{2}+C_{3} .
\end{aligned}
$$

Consequently, it follows that Lemma 3.12 holds with

$$
M_{1}:=\max \left\{\zeta-(\rho-\bar{n}) \log \left\{1-\frac{1}{\sqrt{\bar{n}}}\right\}+\iota \bar{n} \log \bar{n}+C_{2}+C_{3}, \Phi_{\bar{n}}\left(x^{0}, s^{0}\right)\right\} .
$$

Lemma 3.13. Let $M_{1}$ be a fixed constant, and

$$
\Phi_{\bar{n}}\left(x^{k}, s^{k}\right) \leq M_{1} \text { for all } k .
$$

Under the hypothesis of Lemma 3.12. Then, there exists a fixed $M_{2}$ (independent of k) such that

$$
\frac{\min \left\{x_{j}^{k} s_{j}^{k}\right\}}{\left(x^{k}\right)^{T} s^{k}} \geq M_{2}
$$

Proof. As usual, the index $k$ is dropped for simplification. Using the result in Lemma 3.12, we have

$$
M_{1} \geq \Phi_{\bar{n}}(x, s)=(\bar{n} / 2) \log \left\{\left(x^{T} s\right)^{2}+\|r\|^{2}\right\}-\sum_{j=1}^{\bar{n}} \log x_{j} s_{j} \geq \bar{n} \log x^{T} s-\sum_{j=1}^{\bar{n}} \log x_{j} s_{j} .
$$


Hence, it follows that

$$
\begin{aligned}
\log x^{T} s-\log x_{i} s_{i} & \leq M_{1}-(\bar{n}-1) \log x^{T} s+\sum_{j \neq i} \log x_{j} s_{j} \\
& \leq M_{1}-(\bar{n}-1) \log \left\{x^{T} s-x_{i} s_{i}\right\}+\sum_{j \neq i} \log x_{j} s_{j} \\
& \leq M_{1}-(\bar{n}-1) \log \{\bar{n}-1\}=:-\log M_{2}
\end{aligned}
$$

Now let

$$
\begin{aligned}
\sigma(x) & :=\left\{j \in\{1,2, \ldots, \bar{n}\}: x_{j} \geq s_{j}\right\} \text { and } \\
\sigma(s) & :=\left\{j \in\{1,2, \ldots, \bar{n}\}: s_{j} \geq x_{j}\right\} .
\end{aligned}
$$

Combining Lemmas 3.12 and 3.13, the following theorem shows that the sequence $\left\langle x^{k}, s^{k}\right\rangle$ generates a maximal complementary solution for HMCP in the limit.

THEOREM 3.14. Under the hypothesis of Lemma 3.12, the limit point of the sequence $\left\langle x^{k}, s^{k}\right\rangle$ is a maximal complementary solution for HMCP.

Proof. Let $\left(x^{*}, s^{*}\right)$ be any maximal complementary solution for HMCP such that

$$
s^{*}=F\left(x^{*}\right) \text { and }\left(x^{*}\right)^{T} s^{*}=0 .
$$

Let $\left(x^{\infty}, s^{\infty}\right)$ be a limit point of the sequence $\left\langle x^{k}, s^{k}\right\rangle$. In the following we show that $\left(x^{\infty}, s^{\infty}\right)$ is a maximal complementary solution, that is, $\sigma\left(x^{\infty}\right)=\sigma\left(x^{*}\right)$ and $\sigma\left(s^{\infty}\right)=\sigma\left(s^{*}\right)$. Let $|r|^{T}:=\left(\left|r_{1}\right|, \ldots,\left|r_{n}\right|\right)$ and $C_{4}:=\mathcal{O}(1) \frac{\left|r^{0}\right|^{T} x^{*}}{\left(x^{0}\right)^{T} s^{0}} \geq 0$. Using Assumption 3.1, we have

$$
\begin{aligned}
r^{T} x^{*} & \leq|r|^{T} x^{*} \\
& \leq O(1)\left(x^{T} s\right) \frac{\left|r^{0}\right|^{T} x^{*}}{\left(x^{0}\right)^{T} s^{0}} \\
& =C_{4} x^{T} s .
\end{aligned}
$$

From the monotonicity of $F(x)$, for any positive solution $(x, s)>0$, we have

$$
\begin{aligned}
& \left(x-x^{*}\right)^{T}\left(F(x)-F\left(x^{*}\right)\right) \geq 0, \\
\Rightarrow & -x^{T} s^{*}-(s-r)^{T} x^{*} \geq 0, \\
\Rightarrow & -x^{T} s^{*}+s^{T} x^{*} \leq r^{T} x^{*} \leq C_{4} x^{T} s, \quad(\text { using (3.29)) } \\
\Rightarrow & \sum_{j \in \sigma\left(x^{*}\right)} x_{j}^{*} s_{j}+\sum_{j \in \sigma\left(s^{*}\right)} s_{j}^{*} x_{j} \leq C_{4} x^{T} s .
\end{aligned}
$$

As a result, if $j \in \sigma\left(x^{*}\right)$, then

$$
C_{4} x^{T} s \geq s_{j} x_{j}^{*} \geq\left(s_{j} x_{j}\right) \frac{x_{j}^{*}}{x_{j}} \geq \min \left\{x_{j} s_{j}\right\} \frac{x_{j}^{*}}{x_{j}} .
$$

Using Lemma 3.13, the above relation implies

$$
x_{j} \geq \frac{\min \left\{x_{j} s_{j}\right\}}{C_{4} x^{T} s} x_{j}^{*} \geq \frac{M_{2}}{C_{4}} x_{j}^{*} .
$$

Hence, we have $x_{j}^{\infty} \geq\left(M_{2} / C_{4}\right) x_{j}^{*}>0$, consequently $\sigma\left(x^{*}\right)=\sigma\left(x^{\infty}\right)$. The proof of $\sigma\left(s^{*}\right)=\sigma\left(s^{\infty}\right)$ can be constructed in a similar way. 
4. Implementation details. In this section we present the implementation details of a potential reduction homogeneous algorithm for the MCP using the potential function given in Section 3. We describe the details in the context of solving a general convex optimization problem $(\mathrm{CP})$.

4.1. Homogeneous formulation for convex programming. Consider a primal CP

$$
\begin{aligned}
\min & c(x) \\
\text { s.t. } & a_{i}(x) \geq 0, i=1, \ldots, \hat{m}, \\
& a_{i}(x)=0, i=\hat{m}+1, \ldots, m, \\
& \hat{x} \geq 0, \text { and } \tilde{x} \text { is free, }
\end{aligned}
$$

where $x \in R^{n}$ represents the primal variables. We let $\hat{n}+\tilde{n}=n$ and let $x=$ $(\hat{x} ; \tilde{x}) \in R^{\hat{n}} \times R^{\tilde{n}}$, where $\hat{x}$ and $\tilde{x}$ respectively represent the "normal" and "free" primal variables. Here, the function $c(\cdot): R^{n} \rightarrow R$ is convex, the component functions $a_{i}(\cdot): R^{n} \rightarrow R, i=1, \ldots, \hat{m}$ are concave, and the component functions $a_{i}(\cdot): R^{n} \rightarrow$ $R, i=\hat{m}+1, \ldots, m$ are affine.

We let $m=\hat{m}+\tilde{m}$ and $y=(\hat{y} ; \tilde{y}) \in R^{\hat{m}} \times R^{\tilde{m}}$, where $\hat{y}$ and $\tilde{y}$ respectively represent the "normal" and "free" dual variables. Let $s \in R^{\hat{n}}$ represent the vector of dual slacks. Let $\omega:=(x ; y ; \tau ; z ; s ; \kappa)$. In the following we use the notation

$$
\begin{aligned}
\stackrel{\Omega}{\Omega}_{x} & :=\left\{x=(\hat{x} ; \tilde{x}): \hat{x} \in R_{++}^{\hat{n}}, \tilde{x} \in R^{\tilde{n}}\right\}, \\
\check{\Omega}_{y} & :=\left\{y=(\hat{y} ; \tilde{y}): \hat{y} \in R_{++}^{\hat{m}}, \tilde{y} \in R^{\tilde{m}}\right\}, \\
\stackrel{\Omega}{\Omega} & :=\left\{\omega: \omega \in \stackrel{\Omega}{x}_{x} \times \stackrel{\Omega}{\Omega}_{y} \times R_{++}^{\hat{m}+\hat{n}+2}\right\} .
\end{aligned}
$$

Moreover, we assume that functions $c(\cdot)$ and $a_{i}(\cdot), i=1, \ldots, \hat{m}$, are at least twice differentiable on the set $\Omega_{x}$, and the matrix

$$
\left[\begin{array}{cc}
{\left[\begin{array}{cc}
D_{z} & 0 \\
0 & 0
\end{array}\right]} & \nabla a(x) \\
\nabla a(x)^{T} & -\nabla_{x}^{2} \mathcal{L}(x, y)-\left[\begin{array}{cc}
D_{x} & 0 \\
0 & 0
\end{array}\right]
\end{array}\right]
$$

is nonsingular for any positive semidefinite matrices $D_{x} \in R^{\hat{n} \times \hat{n}}$ and $D_{z} \in R^{\hat{m} \times \hat{m}}$.

Here

$$
\mathcal{L}(x, y):=c(x)-y^{T} a(x)
$$

denotes the Lagrangian function, and we use the notation

$$
\nabla_{x} \mathcal{L}(x, y):=\left(\frac{\partial \mathcal{L}(x, y)}{\partial x_{1}}, \ldots, \frac{\partial \mathcal{L}(x, y)}{\partial x_{n}}\right)
$$

to denote the gradient vector of $\mathcal{L}(x, y)$ associated with $x$, and use

$$
\nabla_{x}^{2} \mathcal{L}(x, y):=\left[\begin{array}{ccc}
\frac{\partial^{2} \mathcal{L}(x, y)}{\partial x_{1}^{2}} & \cdots & \frac{\partial^{2} \mathcal{L}(x, y)}{\partial x_{1} \partial x_{n}} \\
\vdots & \ddots & \vdots \\
\frac{\partial^{2} \mathcal{L}(x, y)}{\partial x_{n} \partial x_{1}} & \cdots & \frac{\partial^{2} \mathcal{L}(x, y)}{\partial x_{n}^{2}}
\end{array}\right]
$$


to denote the Hessian matrix of $\mathcal{L}(x, y)$ associated with $x$.

A dual problem associated with (4.1) is

$$
\begin{aligned}
\min & \mathcal{L}(x, y)-\hat{x}^{T} s=\mathcal{L}(x, y)-\nabla_{x} \mathcal{L}(x, y) x \\
\text { s.t. } & \nabla_{x} \mathcal{L}(x, y)^{T}=(s ; 0), \\
& \hat{x}, \hat{y}, s \geq 0, \text { and } \tilde{x}, \tilde{y} \text { are free. }
\end{aligned}
$$

Using (4.1) and (4.2), one can construct optimality conditions to (4.1) as

$$
\begin{aligned}
& \nabla_{x} \mathcal{L}(x, y)^{T}=(s ; 0), \\
& a(x)=(z ; 0), \\
& 0 \leq(s, \hat{y}) \perp(\hat{x}, z) \geq 0, \\
& \tilde{x}, \tilde{y} \text { are free. }
\end{aligned}
$$

Here $z \in R^{\hat{m}}$ represents the vector of surplus variables corresponding to the concave functions $a_{i}(\cdot), i=1, \ldots, \hat{m}$.

Problem (4.3) is a monotone complementarity problem with equality constraints and free variables. Following Andersen and Ye [11], one can formulate a homogenized monotone complementarity problem for (4.3) as follows:

$$
\begin{aligned}
& \tau \nabla_{x} \mathcal{L}(x / \tau, y / \tau)^{T}=(s ; 0), \\
& \tau a(x / \tau)=(z ; 0), \\
& -x^{T} \nabla_{x} \mathcal{L}(x / \tau, y / \tau)^{T}-y^{T} a(x / \tau)=\kappa, \\
& 0 \leq(s, \hat{y}, \kappa) \perp(\hat{x}, z, \tau) \geq 0, \\
& \tilde{x}, \tilde{y} \text { are free, }
\end{aligned}
$$

where $\tau$ and $\kappa$ are introduced homogeneous variables.

Given a point $\omega \in \Omega$, let us define the residual vectors as

$$
\begin{aligned}
& r v_{d}:=\tau g-(s ; 0) \\
& r v_{p}:=\tau a(x / \tau)-(z ; 0), \\
& r v_{g}:=-\kappa-x^{T} g-y^{T} a(x / \tau),
\end{aligned}
$$

and

$$
\begin{aligned}
J & :=\nabla a(x / \tau), \\
g & :=\nabla_{x} \mathcal{L}(x / \tau, y / \tau)^{T}=\nabla c(x / \tau)^{T}-J^{T}(y / \tau) .
\end{aligned}
$$

Let

$$
\left(r_{p} ; r_{d} ; r_{g}\right):=\eta r:=-\eta\left(r v_{p} ; r v_{d} ; r v_{g}\right) .
$$

Let $\hat{X}:=\operatorname{diag}(\hat{x})$ and $\hat{Y}:=\operatorname{diag}(\hat{y})$. Starting from $\omega$, the (perturbed-)Newton method solves the following system of linear equations to compute direction $d_{\omega}:=$ $\left(d_{x} ; d_{z} ; d_{\tau} ; d_{y} ; d_{s} ; d_{\kappa}\right)$ :

$$
\left[\begin{array}{ccc}
H & v_{2} & -J^{T} \\
v_{1}^{T} & H_{g} & -v_{3}^{T} \\
J & v_{3} & 0
\end{array}\right]\left(\begin{array}{c}
d_{x} \\
d_{\tau} \\
d_{y}
\end{array}\right)-\left(\begin{array}{c}
\left(d_{s} ; 0\right) \\
d_{\kappa} \\
\left(d_{z} ; 0\right)
\end{array}\right)=\left(\begin{array}{c}
r_{d} \\
r_{g} \\
r_{p}
\end{array}\right),
$$


and

$$
\begin{aligned}
\hat{X} d_{s}+S d_{\hat{x}} & =r_{\hat{x} s}:=\gamma \mu(\omega) e-\hat{X} s, \\
Z d_{\hat{y}}+\hat{Y} d_{z} & =r_{z \hat{y}}:=\gamma \mu(\omega) e-Z \hat{y}, \\
\tau d_{\kappa}+\kappa d_{\tau} & =r_{\tau \kappa}:=\gamma \mu(\omega)-\tau \kappa,
\end{aligned}
$$

where

$$
\begin{aligned}
\mu(\omega) & :=\left((\hat{x} ; z ; \tau)^{T}(s ; \hat{y} ; \kappa)\right) /(\hat{n}+\hat{m}+1), \\
H & :=\nabla_{x}^{2} \mathcal{L}(x / \tau, y / \tau), \\
v_{1} & :=-\nabla c(x / \tau)^{T}-H(x / \tau), \\
v_{2} & :=\nabla c(x / \tau)^{T}-H(x / \tau), \\
v_{3} & :=a(x / \tau)-J(x / \tau), \\
H_{g} & :=(x / \tau)^{T} H(x / \tau) .
\end{aligned}
$$

We can verify that

$$
\left[\begin{array}{ccc}
H & v_{2} & -J^{T} \\
v_{1}^{T} & H_{g} & -v_{3}^{T} \\
J & v_{3} & 0
\end{array}\right]
$$

is a positive semidefinite matrix. Hence, the complementarity problem (4.4) is monotone.

The coefficient matrix in the system of linear equations (4.5) and (4.6) in general is sparse. By performing simple block elimination on the linear system, we obtain the following alternative formulation, which is an augmented system form [14] with an additional row and column:

$$
\left[\begin{array}{ccc}
D_{z} & J & v_{3} \\
-J^{T} & D_{x} & v_{2} \\
-v_{3}^{T} & v_{1}^{T} & D_{g}
\end{array}\right]\left(\begin{array}{c}
d_{y} \\
d_{x} \\
d_{\tau}
\end{array}\right)=\left(\begin{array}{c}
r_{p}+\left(\hat{Y}^{-1} r_{z \hat{y}} ; 0\right) \\
r_{d}+\left(\hat{X}^{-1} r_{\hat{x} s} ; 0\right) \\
r_{g}+\tau^{-1} r_{\tau \kappa}
\end{array}\right):=\left(\begin{array}{c}
\bar{r}_{p} \\
\bar{r}_{d} \\
\bar{r}_{g}
\end{array}\right)
$$

where

$$
D_{x}:=H+\left[\begin{array}{cc}
\hat{X}^{-1} S & 0 \\
0 & 0
\end{array}\right], \quad D_{z}:=\left[\begin{array}{cc}
Z \hat{Y}^{-1} & 0 \\
0 & 0
\end{array}\right], \quad \text { and } D_{g}:=H_{g}+\tau^{-1} \kappa .
$$

Now define two vectors $\bar{u}:=\left(v_{3} ;-v_{2}\right)$ and $\bar{v}:=\left(-v_{3} ; v_{1}\right)$, and a matrix $M:=$ $\left[\begin{array}{cc}D_{z} & J \\ J^{T} & -D_{x}\end{array}\right]$. Solving (4.7) is equivalent to solving the following two augmented systems:

$$
\begin{aligned}
M q & =\left(\bar{r}_{p} ; \bar{r}_{d}\right) \quad \text { and } \\
M p & =\bar{u} .
\end{aligned}
$$

Therefore, we have obatined the following components of the step direction:

$$
\begin{aligned}
d_{\tau} & =\frac{\bar{r}_{g}-\bar{v}^{T} q}{D_{g}-\bar{v}^{T} p} \quad \text { and } \\
\left(d_{y} ; d_{x}\right) & =q-p d_{\tau}
\end{aligned}
$$

The remaining components $d_{s}, d_{z}, d_{\kappa}$ of the step direction can be easily recoverd. 
$M$ in (4.8) is a symmetric and indefinite matrix. To solve this system we can use the Bunch-Parlett symmetric matrix factorization method [14] or the quasi-definite method [37]. The Bunch-Parlett method is known to avoid numerical instability associated with free variables. In the current test results iOptimize uses the sparse symmetric indefinite matrix factorization library MA57 [16].

4.2. Primal dual updates. Andersen and Ye [11] consider two types of updates: a linear update and a nonlinear update. Let $\omega=(x ; y ; \tau ; z ; s ; \kappa) \in \Omega$ be a feasible solution and $d_{\omega}=\left(d_{x} ; d_{y} ; d_{\tau} ; d_{z} ; d_{s} ; d_{\kappa}\right)$ be a search direction. The linear update computes a new solution $\omega^{+}$as the following:

$$
\omega^{+}:=\omega+\alpha d_{\omega}
$$

where $\alpha \geq 0$ is a given step size. The nonlinear update is a direct result of (2.6). As shown in Lemma 2.3, with $\gamma=1-\eta$ the nonlinear update of the dual variables tend to reduce the infeasibilities and the complementarity gap at the same rate, which is theoretically desirable.

Andersen and Ye [11] propose a merit function to measure the progress in their algorithm. If the merit function is reduced to zero, then a complementary solution is obtained. They show that for a suitably choice of $\gamma$, the (perturbed-) Newton direction is a descent direction for the merit function.

In our implementation we use the potential function (3.1) to evaluate the progress in the algorithm. Given a search direction and a step size, we compute two trial solutions using the linear and nonlinear updates. We accept the trial solution that reduces the potential function more. We give a pseudo code for updating a solution, and discuss the step size computations in iOptimize in the next section.

4.3. Step size computations. To find a controllable step size, Andersen and Ye employ a simple backtracking line search method equipped with safeguards. They require their merit function to be reduced by checking the Goldstein-Armijo rule.

To avoid line search computations, some standard implementations have used a certain fixed distance (step factor) to the boundary. However, a fixed factor $>$ 0.99 may be overly aggressive in the earlier phase of IPM. Mehrotra [25] proposed a heuristic to compute the step factor adaptively. His method adaptively allows for larger (and smaller) step factor values. In our implementation we employ this heuristic to compute step sizes in the primal and dual spaces, and use them to update the point with linear or nonlinear update. This heuristic does not guarantee the reduction of the potential function value (assuming $\nabla_{x} \mathcal{L}(x, y)$ satisfies a SLC). In such a case, we choose the theoretical descent direction from solving (4.5) and (4.6) with suitable parameters $\eta$ and $\gamma$ that guarantees the reduction of the potential function value, and employ a golden-section line search to find a controllable step size (See Section 4.5 for a detailed strategy).

Procedure computeStepFactor gives a pseudo code for computing primal $\left(\sigma_{p}\right)$ and dual $\left(\sigma_{d}\right)$ step factors, Procedure computeStepSize for computing the step size $(\alpha)$ in the primal and dual spaces, and Procedure updateSolution for updating a given point $\omega$ along a search direction $d_{\omega}$ using linear and nonlinear updates. In procedure computeStepFactor, two parameters, $\theta_{l}$ (lower bound) and $\theta_{u}$ (upper bound), are used to safeguard against very tiny or overly aggressive step factors.

4.4. Predictor-corrector technique. The computation of a search direction from (4.5) and (4.6) involves a matrix factorization step and subsequent solves with the factored matrix. The cost of the solves in general is much cheaper, and thus 
Procedure $2\left(\sigma_{p}, \sigma_{d}\right)=$ computeStepFactor $\left(\omega, d_{\omega}\right)$

Input: Current point $\omega=(x ; y ; \tau ; z ; s ; \kappa) \in \stackrel{\Omega}{\Omega}$, search direction $d_{\omega}=$ $\left(d_{x} ; d_{y} ; d_{\tau} ; d_{z} ; d_{s} ; d_{\kappa}\right)$ parameters $\theta_{l}$ and $\theta_{u}$.

Output: Primal step factor $\sigma_{p}$ and dual step factor $\sigma_{d}$.

1: Find the blocking variables:

$$
\begin{aligned}
l x & :=\arg \min _{j=1, \ldots, \hat{n}}\left\{-x_{j} /\left(d_{x}\right)_{j} \text { if }\left(d_{x}\right)_{j}<0\right\}, \\
l s & :=\arg \min _{j=1, \ldots, \hat{n}}\left\{-s_{j} /\left(d_{s}\right)_{j} \text { if }\left(d_{s}\right)_{j}<0\right\}, \\
l z & :=\arg \min _{i=1, \ldots, \hat{m}}\left\{-z_{i} /\left(d_{z}\right)_{i} \text { if }\left(d_{z}\right)_{i}<0\right\}, \\
l y & :=\arg \min _{i=1, \ldots, \hat{m}}\left\{-y_{i} /\left(d_{y}\right)_{i} \text { if }\left(d_{y}\right)_{i}<0\right\}, \\
l \tau & :=-\tau / d_{\tau} \text { if } d_{\tau}<0, \\
l \kappa & :=-\kappa / d_{\kappa} \text { if } d_{\kappa}<0 .
\end{aligned}
$$

2: Compute an estimate of the duality gap:

$$
\begin{aligned}
\mu_{x} & :=\sum_{j=1}^{\hat{n}}\left(x_{j}+\left(d_{x}\right)_{j}\right)\left(s_{j}+\left(d_{s}\right)_{j}\right), \\
\mu_{z} & :=\sum_{i=1}^{\hat{m}}\left(z_{i}+\left(d_{z}\right)_{i}\right)\left(y_{i}+\left(d_{y}\right)_{i}\right), \\
\mu_{\tau} & :=\left(\tau+d_{\tau}\right)\left(\kappa+d_{\kappa}\right), \\
\mu & :=\left(\left(\mu_{x}+\mu_{z}+\mu_{\tau}\right)\left(1-\theta_{l}\right)\right) /(\hat{n}+\hat{m}+1) .
\end{aligned}
$$

3: Compute the step factors for all the variables:

$$
\begin{aligned}
\sigma_{x} & :=\left(\frac{\mu}{s_{l x}+\left(d_{s}\right)_{l x}}-x_{l x}\right)\left(\frac{1}{\left(d_{x}\right)_{l x}}\right), \\
\sigma_{s} & :=\left(\frac{\mu}{x_{l s}+\left(d_{x}\right)_{l s}}-s_{l s}\right)\left(\frac{1}{\left(d_{s}\right)_{l s}}\right), \\
\sigma_{z} & :=\left(\frac{\mu}{y_{l z}+\left(d_{y}\right)_{l z}}-z_{l z}\right)\left(\frac{1}{\left(d_{z}\right)_{l z}}\right), \\
\sigma_{y} & :=\left(\frac{\mu}{z_{l y}+\left(d_{z}\right)_{l y}}-y_{l y}\right)\left(\frac{1}{\left(d_{y}\right)_{l y}}\right), \\
\sigma_{\tau} & :=\left(\frac{\mu}{\kappa_{l \tau}+\left(d_{\kappa}\right)_{l \tau}}-\tau_{l \tau}\right)\left(\frac{1}{\left(d_{\tau}\right)_{l \tau}}\right), \\
\sigma_{\kappa} & :=\left(\frac{\mu}{\tau_{l \kappa}+\left(d_{\tau}\right)_{l \kappa}}-\kappa_{l \kappa}\right)\left(\frac{1}{\left(d_{\kappa}\right)_{l \kappa}}\right) .
\end{aligned}
$$

4: Compute primal and dual step factors:

$$
\begin{aligned}
\sigma_{p} & :=\max \left\{\theta_{l}, \min \left\{\sigma_{x}, \sigma_{z}, \sigma_{\tau}, \theta_{u}\right\}\right\}, \\
\sigma_{d} & :=\max \left\{\theta_{l}, \min \left\{\sigma_{s}, \sigma_{y}, \sigma_{\kappa}, \theta_{u}\right\}\right\} .
\end{aligned}
$$


Procedure $3 \alpha=$ computeStepSize $\left(\omega, d_{\omega}\right)$

Input: Current point $\omega=(x ; z ; \tau ; y ; s ; \kappa) \in \stackrel{\Omega}{\Omega}$ and search direction $d_{\omega}=$ $\left(d_{x} ; d_{z} ; d_{\tau} ; d_{y} ; d_{s} ; d_{\kappa}\right)$.

Output: Primal and dual step size $\alpha$.

1: Compute the step sizes to the boundary

$$
\begin{aligned}
\alpha_{x} & :=\min _{j=1, \ldots, \hat{n}}\left\{-x_{j} /\left(d_{x}\right)_{j} \text { if }\left(d_{x}\right)_{j}<0\right\}, \\
\alpha_{s} & :=\min _{j=1, \ldots, \hat{n}}\left\{-s_{j} /\left(d_{s}\right)_{j} \text { if }\left(d_{s}\right)_{j}<0\right\}, \\
\alpha_{z} & :=\min _{i=1, \ldots, \hat{m}}\left\{-z_{i} /\left(d_{z}\right)_{i} \text { if }\left(d_{z}\right)_{i}<0\right\}, \\
\alpha_{y} & :=\min _{i=1, \ldots, \hat{m}}\left\{-y_{i} /\left(d_{y}\right)_{i} \text { if }\left(d_{y}\right)_{i}<0\right\}, \\
\alpha_{\tau} & :=-\tau / d_{\tau} \text { if } d_{\tau}<0, \\
\alpha_{\kappa} & :=-\kappa / d_{\kappa} \text { if } d_{\kappa}<0 .
\end{aligned}
$$

2: Call Procedure computeStepFactor $\left(\sigma_{p}, \sigma_{d}, \omega, d_{\omega}\right)$ to compute the primal step factor $\left(\sigma_{p}\right)$ and the dual step factor $\left(\sigma_{d}\right)$.

3: Compute primal and dual step sizes:

$$
\begin{aligned}
& \alpha_{p}:=\sigma_{p} \times \min \left\{\alpha_{x}, \alpha_{z}, \alpha_{\tau}\right\}, \\
& \alpha_{d}:=\sigma_{d} \times \min \left\{\alpha_{s}, \alpha_{y}, \alpha_{\kappa}\right\},
\end{aligned}
$$

4: Set $\alpha:=\min \left\{\alpha_{p}, \alpha_{d}\right\}$.

Procedure $4 \omega^{+}=$updateSolution $\left(\omega, d_{\omega}, \alpha\right)$

Input: Current point $\omega=(x ; y ; \tau ; z ; s ; \kappa) \in \stackrel{\Omega}{ }$, search direction $d_{\omega}=$ $\left(d_{x} ; d_{y} ; d_{\tau} ; d_{z} ; d_{s} ; d_{\kappa}\right)$, primal and dual step size $\alpha$.

Output: Updated point $\omega^{+} \in \Omega^{\circ}$.

1: Compute the primal and dual updates:

$\omega^{l}:=\omega+\alpha\left(d_{x} ; d_{y} ; d_{\tau} ; d_{z} ; d_{s} ; d_{\kappa}\right)$.

2: Compute the nonlinear update:

$$
\begin{aligned}
x^{n} & :=x+\alpha d_{x}, \\
y^{n} & :=y+\alpha d_{x}, \\
\tau^{n} & :=\tau+\alpha d_{\tau}, \\
z_{i}^{n} & :=\left((1-\alpha \eta)\left(-r v_{p}\right)_{i}+\tau^{n} a_{i}\left(x^{n} / \tau^{n}\right)\right), i=1, \ldots, \hat{m}, \\
s_{j}^{n} & :=\left((1-\alpha \eta)\left(-r v_{d}\right)_{j}+\tau^{n} \nabla_{x} \mathcal{L}_{j}\left(x^{n} / \tau^{n}, y^{n} / \tau^{n}\right)^{T}\right), j=1, \ldots, \hat{n}, \\
\kappa^{n} & :=\left((1-\alpha \eta)\left(-r v_{g}\right)-\left(x^{n}\right)^{T} \nabla_{x} \mathcal{L}\left(x^{n} / \tau^{n}, y^{n} / \tau^{n}\right)^{T}-\left(y^{n}\right)^{T} a\left(x^{n} / \tau^{n}\right)\right) .
\end{aligned}
$$

3: Check if it is beneficial to take linear update:

4: if $\Phi_{\rho}\left(\omega^{n}\right) \geq \Phi_{\rho}\left(\omega^{l}\right)$ then

5: $\quad$ Set $\omega^{+}:=\omega^{l}$.

6: else

7: $\quad$ Set $\omega^{+}:=\omega^{n}$.

8: end if 
it leads to the idea of reusing the factorization of the linear system to compute a better search direction. Several approaches have been developed based on this idea. For example, Mehrotra corrector [25] and Gondzio's higher-order corrector [18] are proper choices. The presented empirical results are based on using Mehrotra corrector only. We now describe an adaption of this technique for our context.

Let $\omega^{k} \in \Omega$ be the current point, we first compute an affine (pure Newton) direction by solving (4.5) and (4.6) with $\eta=1$ and $\gamma=0$, that is,

$$
\begin{aligned}
& r_{p}:=-r v_{u}^{k}, r_{\hat{x} s}:=-\hat{X}^{k} s^{k}, \\
& r_{d}:=-r v_{p}^{k}, r_{z \hat{y}}:=-Z^{k} \hat{y}^{k}, \\
& r_{g}:=-r v_{d}^{k}, r_{\tau \kappa}:=-\tau^{k} \kappa^{k} .
\end{aligned}
$$

The resulting direction is denoted by $d_{\omega}^{a}=\left(d_{x}^{a} ; d_{z}^{a} ; d_{\tau}^{a} ; d_{y}^{a} ; d_{s}^{a} ; d_{\kappa}^{a}\right)$. Next we compute a step size $\alpha^{a}$ by calling Procedure $\alpha^{a}=$ computeStepSize $\left(\omega^{k}, d_{\omega}^{a}\right)$ where $\omega^{k}$ and $d_{\omega}^{a}$ are the input, and $\alpha^{a}$ is the output, and then compute a tentative point $\omega^{a}$ by calling Procedure $\omega^{k}=$ updateSolution $\left(\omega^{a}, d_{\omega}^{a}, \alpha^{a}\right)$ where $\omega^{k}, d_{\omega}$, and $\alpha^{a}$ are the input and $\omega^{a}$ is the output.

Mehrotra suggested to determine the barrier parameters $\gamma^{a}$ and $\eta^{a}$ by using the predicted reduction in the complementarity gap $\mu\left(\omega^{a}\right)$ along the affine direction. The idea behind this heuristic is to reduce the centrality parameter $\gamma^{a}$ more when the IPM can make good progress towards optimality in the affine direction. In our implementation we compute the barrier parameters $\gamma^{a}$ and $\eta^{a}$ as follows:

$$
\begin{aligned}
& \gamma^{a}=\min \left\{0.99, \max \left\{\left(\frac{\mu\left(\omega^{a}\right)}{\mu\left(\omega^{k}\right)}\right)^{3}, 0.01\right\}\right\}, \\
& \eta^{a}=1-\gamma^{a} .
\end{aligned}
$$

The new complementarity gap is given by

$$
\begin{aligned}
\left(\hat{X}^{k}+D_{\hat{x}}^{a}\right)\left(s^{k}+d_{s}^{a}\right) & =D_{\hat{x}}^{a} d_{s}^{a}, \\
\left(Z^{k}+D_{z}^{a}\right)\left(\hat{y}^{k}+d_{\hat{y}}^{a}\right) & =D_{z}^{a} d_{\hat{y}}^{a}, \\
\left(\tau^{k}+d_{\kappa}^{a}\right)\left(\kappa^{k}+d_{\tau}^{a}\right) & =d_{\tau}^{a} d_{\kappa}^{a},
\end{aligned}
$$

where $D_{\hat{x}}^{a}:=\operatorname{diag}\left(d_{\hat{x}}^{a}\right)$ and $D_{z}^{a}:=\operatorname{diag}\left(d_{z}^{a}\right)$. Since the new complementarity product ideally should be equal to $\mu\left(\omega^{a}\right) e$, the new search direction compensates for the error and thus is computed with the following right-hand side:

$$
\begin{aligned}
& r_{p}:=-\eta^{a} r v_{p}, r_{\hat{x} s}:=\gamma^{a} \mu\left(\omega^{a}\right) e-\hat{X}^{k} s^{k}-D_{\hat{x}}^{a} d_{s}^{a}, \\
& r_{d}:=-\eta^{a} r v_{d}, r_{z \hat{y}}:=\gamma^{a} \mu\left(\omega^{a}\right) e-Z^{k} \hat{y}^{k}-D_{z}^{a} d_{\hat{y}}^{a}, \\
& r_{g}:=-\eta^{a} r v_{g}, r_{\tau \kappa}:=\gamma^{a} \mu\left(\omega^{a}\right)-\tau^{k} \kappa^{k}-d_{\tau}^{a} d_{\kappa}^{a} .
\end{aligned}
$$

The resulting direction here is called "Mehrotra direction" and is denoted by $d_{\omega}^{m}$.

4.5. Search guided by the potential function. The implementation logic in iOptimize that combines the predictor and corrector search directions at each iteration is now given. At the core of our implementation is to ensure sufficient reduction in the potential function at each iteration, while using Mehrotra direction to further improve reduction in the potential function.

At each iteration $k$ we first check if $\Phi_{\hat{n}+\hat{m}+1}\left(\omega^{k}\right) \leq \iota(\hat{n}+\hat{m}+1) \log (\hat{n}+\hat{m}+1)$. This safeguard is used to make sure that our algorithm generates a maximal complementary solution. If it is not satisfied, then we use the theoretical direction computation phase; otherwise we compute a Mehrotra direction $d_{\omega}^{m}$ and use it to generate a tentative step 
size $\omega^{m}$ by calling Procedure $\alpha^{m}=$ computeStepSize $\left(\omega^{k}, d_{\omega}^{m}\right)$, where $\omega^{k}$ and $d_{\omega}^{m}$ are the input, and $\alpha^{m}$ is the output. Then we compute a tentative point $\omega_{m}$ by calling Procedure $\omega^{m}=$ updateSolution $\left(d_{\omega}^{m}, \omega^{k}, \alpha^{m}\right)$, where $\omega^{k}, d_{\omega}^{m}$, and $\alpha^{m}$ are the input and $\omega^{m}$ is the output. Though $d_{\omega}^{m}$ generally enhances the convergence, it does not guarantee reduction of the potential function value, or maintain the overall decrease in the infeasibility to complementarity ratio (Assumption 3.1). Hence we determine the quality of the Mehrotra direction by evaluating the potential function value at $\omega^{m}$ and check if $\omega^{m}$ satisfies Assumption 3.1. We note that checking 3.1(ii) requires the knowledge of the scaled Lipschitz constant, which may not be available in practice; hence, we simply check if $\mu\left(\omega^{m}\right)<\mu\left(\omega^{k}\right)$. If $\Phi_{\rho}\left(\omega^{m}\right)<\Phi_{\rho}\left(\omega^{k}\right)$ and Assumption 3.1 is satisfied, we set $\omega^{k+1}:=\omega^{m}$; otherwise we compute a theoretical direction $d_{\omega}^{t}$ from (4.5) and (4.6), and iteratively employs a golden-section linear search method [13] to find a controllable step size $\alpha^{t}$, and use it to compute a new tentative point $\omega^{t}$ by calling Procedure $\omega^{t}=$ updateSolution $\left(\omega^{k}, d_{\omega}^{t}, \alpha\right)$, where $\omega^{k}, d_{\omega}^{t}$, and $\alpha^{t}$ are the input and $\omega^{t}$ is the output. We terminate the line search if the potential function achieves sufficient decrease.

Note that if the scaled Lipschitz constant is large, then many function evaluations may be needed in the golden section line search. To avoid this we simply take the step after 4 iterations of golden section method, and monitor the progress of the potential function over multiple interior point iterates. We ensure that the potential function is eventually reduced over these multiple iterates.

A flowchart of the implementation of search direction computations is given in Figure 4.1.

4.6. Starting point and termination criteria. Andersen and Ye [11] show that the HMCP IPM achieves consistently good performance even for a trivial starting point

$$
\left(\hat{x}^{0} ; \tilde{x}^{0} ; \hat{y}^{0} ; \tilde{y}^{0} ; \tau^{0} ; z^{0} ; s^{0} ; \kappa^{0}\right)=(e ; 0 ; e ; 0 ; 1 ; e ; e ; 1) .
$$

This trivial starting point is used in the results reported in this paper. However, it might be possible to further improve the computational results by implementing a (better) non-trivial starting point.

Another important issue is the termination criteria. We choose the termination criteria used by Andersen and Ye [11] with slight modifications. Define the primal and dual objective values by

$$
\operatorname{PriObj}:=c(x / \tau), \text { and DualObj }:=\mathcal{L}(x / \tau, y / \tau)-\nabla_{x} \mathcal{L}(x / \tau, y / \tau)(x / \tau) .
$$

Let $r v_{p}^{k}, r v_{d}^{k}$, and $r v_{g}^{k}$ be the vectors of the residuals corresponding to the $k$ th iterate. We terminate the algorithm whenever one of the following criteria is satisfied:

- For QP, an (approximate) optimal solution is obtained if

$$
\begin{array}{r}
\frac{\left\|r v_{p}^{k}\right\|}{\tau\{1+\|b\|\}}<10^{-8}, \\
\frac{\left\|r v_{d}^{k}\right\|}{\tau\{1+\|c\|\}}<10^{-8}, \\
\min \left\{\frac{\mid \text { DualObj }- \text { PriObj| }}{1+\mid \text { DualObj| }}, \frac{\left|r v_{g}^{k}\right|}{1+\left|r v_{g}^{0}\right|}\right\}<10^{-8} .
\end{array}
$$




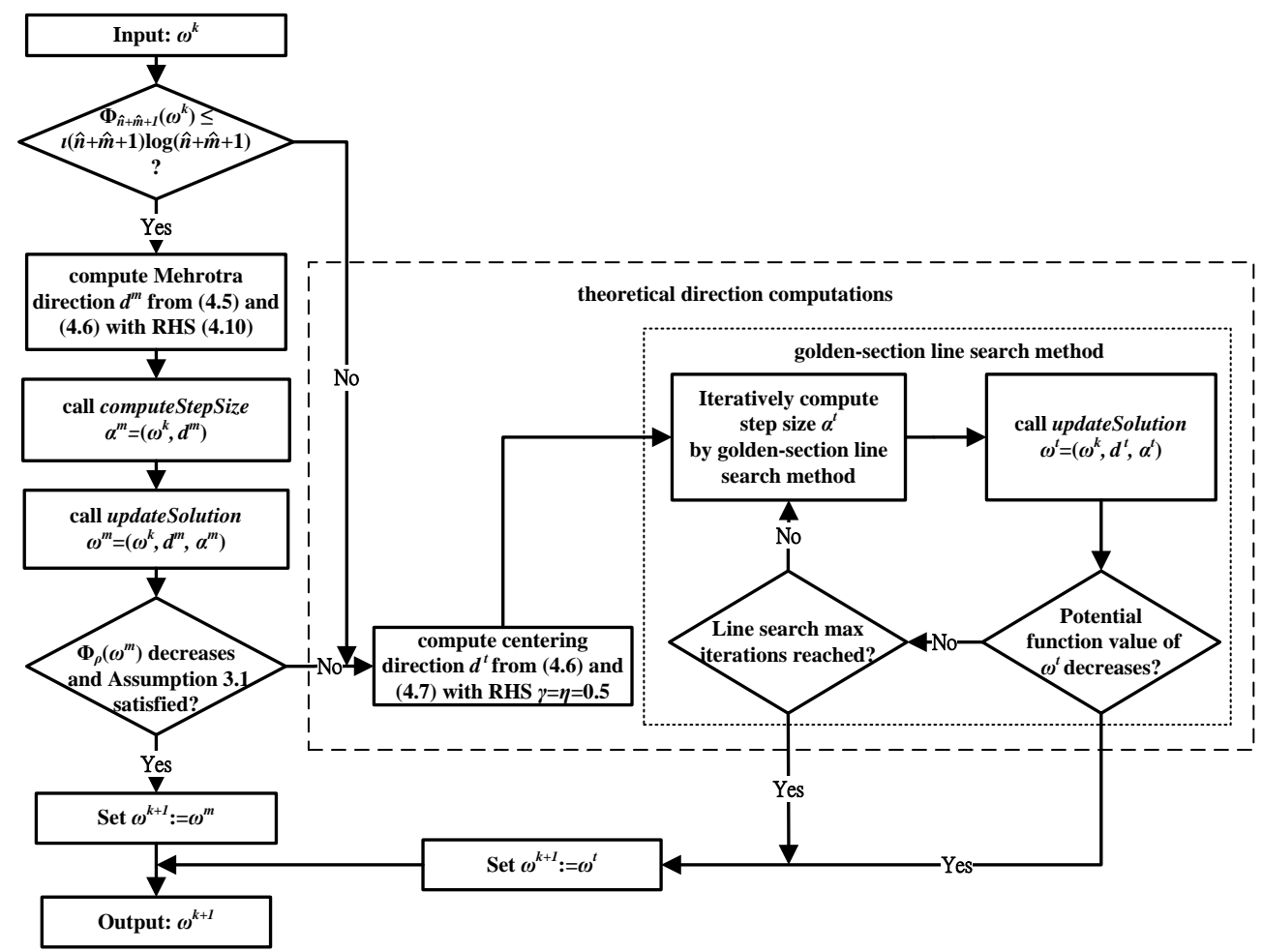

Fig. 4.1: Implementation of search direction computations

- For QCQP and CP, an (approximate) optimal solution is obtained if

$$
\begin{gathered}
\frac{\left\|r v_{p}^{k}\right\|}{\max \left\{1,\left\|r v_{p}^{0}\right\|\right\}}<10^{-8}, \\
\frac{\left\|r v_{d}^{k}\right\|}{\max \left\{1,\left\|r v_{d}^{0}\right\|\right\}}<10^{-8}, \\
\frac{\left|r v_{g}^{k}\right|}{\max \left\{1,\left|r v_{g}^{0}\right|\right\}}<10^{-6} .
\end{gathered}
$$

- For QP, QCQP, and CP, the problem is (near) infeasible if

$$
\mu\left(\omega^{k}\right)<\mu\left(\omega^{0}\right) 10^{-8} \text { and } \frac{\tau^{k}}{\min \left\{1, \kappa^{k}\right\}}<\frac{\tau^{0}}{\kappa^{0}} 10^{-12} .
$$

We note that Andersen [9] developed an infeasibility certificate for the homogeneous monotone complementarity problems, that provide further information about whether the primal or dual problem is infeasible. This feature, however, has not been implemented in the current version of iOptimize.

5. Computational results. iOptimize was compiled with the Visual Studio 2010 compiler. All computations were performed on a $3.2 \mathrm{GHz}$ Intel Dual-Core CPU machine with $4 \mathrm{~GB}$ RAM. The program is run on one CPU only. An AMPL [1] interface 


\begin{tabular}{lll}
\hline Parameter & Value & Explanation of the parameter \\
\hline$\theta$ & $\hat{n}+\hat{m}+1$ & Parameter in the potential function (3.1) \\
$\rho$ & 100 & Parameter in the potential function (3.1) \\
$\left(\theta_{l}, \theta_{u}\right)$ & $(0.999,1.0)$ & Parameters for QP in Procedure computeStep Factor \\
$\left(\theta_{l}, \theta_{u}\right)$ & $(0.9,1.0)$ & Parameters for QCQP and CP in Procedure computeStepFactor \\
$\iota$ & $(\hat{n}+\hat{m}+1)^{2}$ & Parameter in (3.28) \\
$(\Theta(1), \Omega(1))$ & $\left(10^{-2}, 10^{2}\right)$ & Parameters in Assumption 3.1 \\
\hline
\end{tabular}

Table 5.1: The default parameter settings in iOptimize

is implemented in iOptimize to read the AMPL nonlinear models and the corresponding first and second-derivatives. All problems are solved using the same default parameter settings, which are shown in Table 5.1.

To provide a clearer summary of the results, we use performance profiles [15]. Consider a set $A$ of $n_{a}$ algorithms, a set $P$ of $n_{p}$ problems, and a performance measure $m_{a, p}$, e.g., node count or computation time. We compare the performance on problem $p$ by algorithm $a$ with the best performance by any algorithm on this problem using the following performance ratio

$$
r_{p, a}=\frac{m_{p, a}}{\min \left\{m_{p, a} \mid a \in A\right\}} .
$$

We therefore obtain an overall assessment of the performance of the algorithm by defining the following value

$$
\rho_{a}(\tau)=\frac{1}{n_{p}} \text { size }\left\{p \in P \mid r_{p, a} \leq \tau\right\} .
$$

This represents the probability for algorithm $a$ that the performance ratio $r_{p, a}$ is within a factor $\tau$ of the best possible ratio. The function $\rho_{a}(\cdot)$ represents the distribution function for the performance ratio.

5.1. Computational results on feasible problems. We solve the convex QP problems from Maros and Mézáros's QP test set [24], the convex QCQP problems from Mittelmann's QCQP test set [5] and Vanderbei's AMPL nonlinear Cute and Non-Cute set [2], the general convex CP problems from Vanderbei's AMPL nonlinear test set [2], and from Leyffer's mixed-integer nonlinear test set [6] while ignoring the integrality requirements.

Tables A.1-A.4 give the computational results that are obtained with the default parameter settings. Each row in these tables contains the profiles of the problems before and after presolving, the primal and the dual objectives, the number of iterations (Avg Iters), the computation times (Presolve Time and Solver Time), the proportion of the effort in evaluating the potential function (Prop $\Phi(\%)$ ), and the average number of search directions used (Avg Dirs) (here two means iOptimize uses pure Newton direction and Mehrotra corrector, whereas one means iOptimize uses the theoretical direction only). We note that the QCQP problems in Mittelmann's test set are equivalent to those of Maros and Mézáros's QP test set, as each QP problem is reformulated as a QCQP problem with an additional quadratic constraint and a free variable.

Tables A.5-A.8 provide a comparison between MOSEK homogeneous and self-dual interior point optimizer and iOptimize under their default settings. We focus on the number of iterations needed to achieve the desired accuracy by both solvers. 
Table A.5 gives the computational results for solving 127 QP problems. The average number of iterations used by iOptimize (15.92 iters) and MOSEK (16.22 iters) are comparable. All problems can be solved within 50 iterations by both solvers. Note that for three problems (liswet4, powell20, and qpilotno) MOSEK terminates with NEAR_OPTIMAL status. This implies MOSEK cannot compute a solution that has the prescribed accuracy.

Table A.6 gives the computational results for solving Mittelmann's QCQP test set problems. Here two different average performance results are provided. "Avg1" provides the average performance on problems for which MOSEK terminate with OPTIMAL, NEAR_OPTIMAL or UNKNOWN status. For problems QQ-aug2dqp and QQ-powell20, MOSEK is not able to converge to the optimal solution within 400 iterations. We exclude both problems in "Avg1". For problems QQ-laser, QQ-qforplan, and QQ-qseba, MOSEK requires more than 50 interior point iterations to successfully solve. We recompute the average performance statistics again in "Avg2" by further excluding these three examples. Considering average performances in these experiments, iOptimize (Avg1=16.43 iters, Avg2=16.26 iters) is significantly better than MOSEK (Avg1=20.91 iters, Avg2=18.34 iters). Note that for QQ-liswet1, QQ-liswet7-QQ-liswet12, MOSEK terminates with UNKNOWN status. This may happen when MOSEK converges slowly, and hence the primal, dual, or gap residuals may not attain the prescribed accuracy. On the other hand, iOptimize is able to terminate with OPTIMAL status for all the test problems within 40 iterations.

Table A.7 contains the computational results for solving 11 QCQP problems in the Vanderbei's AMPL nonlinear test set. Comparing the size to those in Mittelmann's QCQP test set, the problems here are relatively small, but may include a quadratic objective term and more than one quadratic constraint. Considering the average iterations required for solving the problems, both solvers generate similar computational results (MOSEK Avg=11.36 iters versus iOptimize Avg=11.64 iters).

Table A.8 contains the computational results for solving 46 general CP problems. Thirty five of the CP problems are selected from Vanderbei's AMPL nonlinear Cute and Non-Cute set, and 11 of the CP problems are selected from Leyffer's mixed-integer nonlinear test set. Two different average performance statistics are provided. "Avg1" provides the average performance on all the instances, except problem dallass, for which MOSEK fails to converge to an optimal solution within 400 iterations. For problems dallasm, dallasl, and elena-s383, MOSEK respectively requires 128,74 , and 85 iterations before terminating with OPTIMAL status. Thus we recompute the average statistics again in "Avg2" by additionally excluding these three problems. Comparing "Avg1", the average number of iterations used by iOptimize (14.00 iters) is better than those used by MOSEK (18.46 iters). By excluding these problems for which MOSEK requires more than 50 iterations to solve ("Avg2"), the performance comparison between both solvers becomes comparable, though iOptimize (12.69 iters) still performs slightly better than MOSEK (13.07 iters). Note that for problems antenna-antenna2, firfilter-fir_convex, and wbv-lowpass2, MOSEK terminates with NEAR_OPTIMAL status.

The average number of directions used by iOptimize is $\approx 1.93$, i.e., Mehrotra direction was not used in about $7 \%$ of the iterations. It implies that the average proportion of the computations for the theoretical direction with a fixed step size is not significant. In these experiments we observed that Assumption 3.1 and the requirement $\Phi_{\hat{n}+\hat{m}+1}\left(\omega^{k}\right) \leq \iota(\hat{n}+\hat{m}+1) \log (\hat{n}+\hat{m}+1)$ is always satisfied over all the iterates, implying that iOptimize uses the theoretical direction only in the case 
where Mehrotra direction fails to reduce the potential function value. We also note that the average proportion of the efforts used for the potential function evaluations is $3.1 \%$, suggesting that the computational demands for evaluating the potential function is also not significant, while a potential reduction algorithm is implemented that guarantees global linear polynomial time convergence.

Figure 5.1 shows the performance profiles for feasible problems comparing the iteration performances of iOptimize and MOSEK.

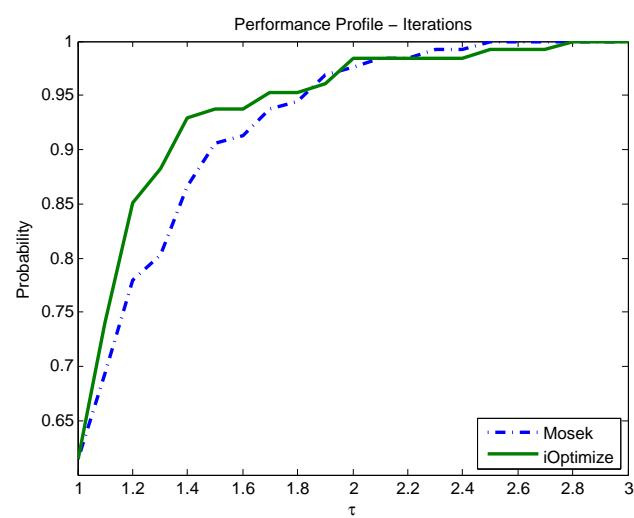

(a) Maros and Mézáros's QP feasible problems

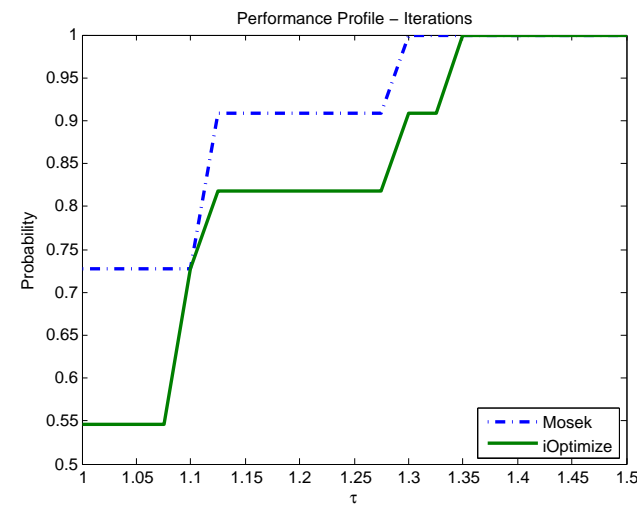

(c) Vanderbei's QCQP feasible problems

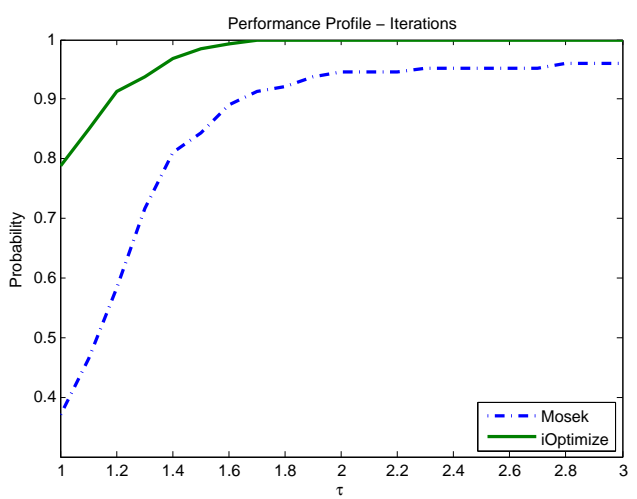

(b) Mittelmann's QCQP feasible problems

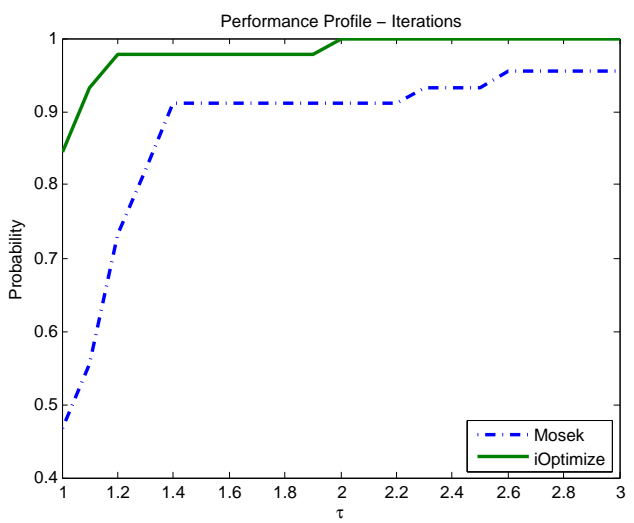

(d) General CP feasible problems

Fig. 5.1: Performance profiles of iOptimize and MOSEK on feasible problems

5.2. Computational results on infeasible problems. In this section we test the performance of iOptimize for solving the QCQP and CP infeasible problems. With the lack of the infeasible test set in the public domain, we create infeasible test problems by adding invalid objective constraints to the feasible problems.

Let $x^{*}$ be an optimal solution of problem (4.1). We create a corresponding infeasible problem that has the form:

$$
\begin{aligned}
\min & c(x) \\
\text { s.t. } & a_{i}(x) \geq 0, i=1, \ldots, m_{n}, \\
& a_{i}(x)=0, i=\hat{m}+1, \ldots, m,
\end{aligned}
$$




$$
\begin{aligned}
& c(x) \leq c\left(x^{*}\right)-\lambda, \\
& \hat{x} \geq 0, \text { and } \tilde{m} \text { is free, }
\end{aligned}
$$

where $\lambda$ is a constant with positive value. We choose

$$
\lambda:= \begin{cases}2\left|c\left(x^{*}\right)\right|, & \text { if }\left|c\left(x^{*}\right)\right| \geq 100 \\ 100, & \text { if }\left|c\left(x^{*}\right)\right|<100 .\end{cases}
$$

Hence, the problems are made significantly infeasible.

Using this construction we create 127 infeasible QCQP problems from Maros and Mézáros's QP test set [24], 11 QCQP infeasible problems from Vanderbei's AMPL nonlinear Cute and Non-Cute set [2], 35 CP infeasible problems from Vanderbei's AMPL nonlinear Cute and Non-Cute set [2], and $11 \mathrm{CP}$ infeasible problems from Leyffer's mixed-integer nonlinear test set [6] without integrality requirements.

Tables A.9-A.11 give the computational results that are obtained with the default parameter settings. The number of iterations (Avg Iters), the computation times (Presolve Time and Solver Time), the average number of search directions used (Avg Dirs), and the proportion of the effort in evaluating the potential function (Prop $\Phi(\%)$ ) are given. Tables A.12-A.14 provide a comparison between MOSEK and iOptimize under their default settings.

We focus on the number of iterations needed to detect infeasibility by both solvers. Table A.12 contains the computational results for solving 127 QCQP infeasible problems created from Maros and Mézáros's QP test set. For 38 problems MOSEK fails to detect infeasibility within 400 iterations. For problems iQQ-qScagr25 and iQQ-qstandat, MOSEK terminates with UNKNOWN status. We compute the average iterations in "Avg1" by excluding these 38 examples. Comparing "Avg1", iOptimize ( 28.15 iters) requires approximately $30 \%$ fewer iterations than MOSEK ( 42.20 iters). This is because MOSEK requires more than 60 iterations to detect the infeasibility in 12 problems. We further exclude these 12 problems and recompute the average iterations in "Avg2". Comparing "Avg2", the performance of iOptimize (28.15 iters) is comparable to that of MOSEK (27.39 iters). However, iOptimize is able to correctly detect infeasibility for each the problem within 50 interior point iterations.

Table A.13 has the computational results for solving the 11 QCQP infeasible problems created from Vanderbei's AMPL nonlinear test set. We provide the average statistics by excluding problem ipolak4, which MOSEK fails to solve within 400 iterations. Though the comparison shows that iOptimize (24.60 iters) requires approximately $20 \%$ more iterations than MOSEK (20.30 iters), iOptimize correctly solves all these infeasible problems.

Table A.14 has the computational results for solving $46 \mathrm{CP}$ infeasible problems created from Vanderbei's AMPL nonlinear Cute and Non-Cute set, and Leyffer's mixed-integer nonlinear test set. For problems idallasl, idallasm, and idallass, MOSEK receives function evaluate error from AMPL. iOptimize receives the same error while solving problem idallasl. In seven problems MOSEK fails to declare the infeasible status within 400 iterations. We compute the average statistics "Avg2" by excluding these 10 problems. Comparing "Avg1", MOSEK (66.33 iters) requires significantly more iterations than iOptimize (19.44 iters). This happens because MOSEK requires more than 60 iterations to detect infeasibility in 13 problems whereas iOptimize needs this in only one problem. We further exclude these 10 problems and recompute the average statistics in "Avg2". Comparing "Avg2", iOptimize (16.04 iters) requires $60 \%$ more iteration than MOSEK (11.74 iters). This is because MOSEK is 
able to detect infeasibility during the preprocessing phase in 11 cases; on the other hand, iOptimize preprocessor does not detect any infeasibility. This highlights the importance of advanced preprocessing implementations, which is not the focus of research described in the current paper.

Note that for problem antenna-iantenna2, iOptimize requires 186 iterations to solve, which is significantly larger than the average iterations (28.07 iters). With a fixed step factor parameter settings $\left(\theta_{l}, \theta_{u}\right)=(0.9,0.9)$, iOptimize can solve this problem in 46 iterations.

Observe that the average number of directions used for this problem is around 1.39 , which is significantly smaller than that used for solving the feasible problems. This suggests the importance of using the theoretical direction and potential function to decide the usefulness of a direction.

Figure 5.2 shows the performance profiles for the infeasible problems comparing the iteration performances of iOptimize and MOSEK. Note that problems which iOptimize or MOSEK fails to solve or solves them during the preprocessing phase are not considered.

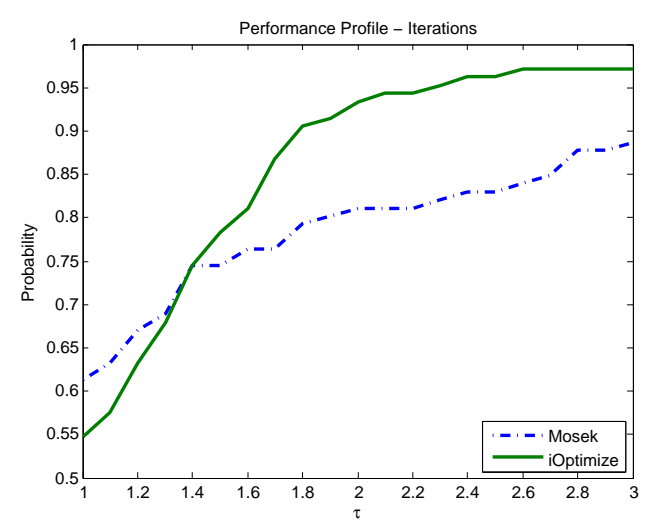

(a) Mittelmann's QCQP infeasible problems

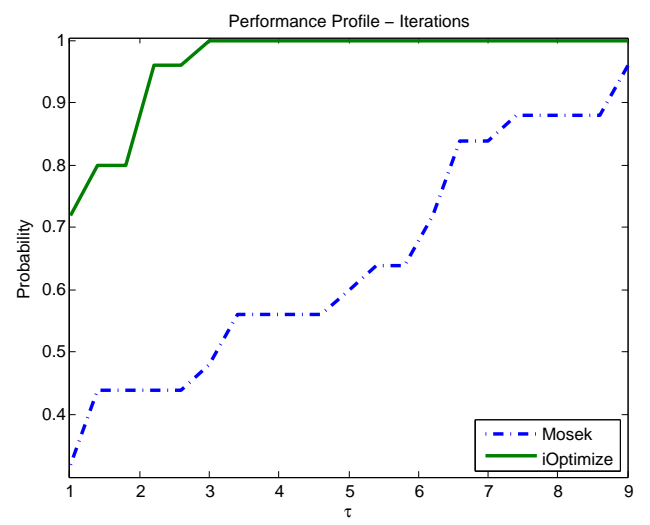

(c) General CP infeasible problems

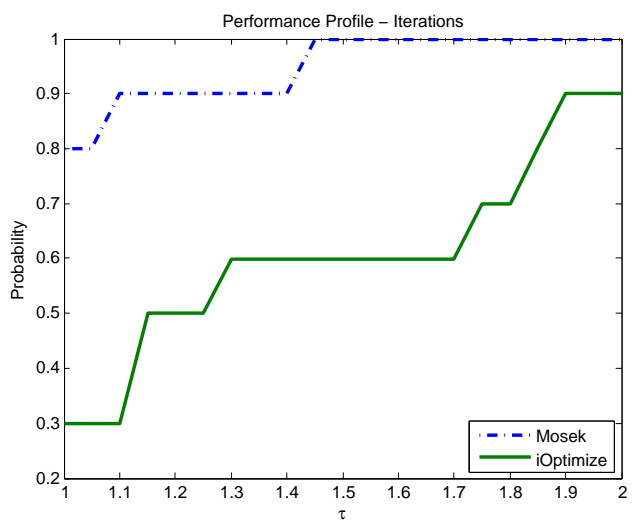

(b) Vanderbei's QCQP infeasible problems

Fig. 5.2: Performance profiles of iOptimize and MOSEK on infeasible problems 
5.3. Computational comparison with general nonlinear solvers. In this section we compare iOptimize with general nonlinear solver Knitro and Ipopt on $\mathrm{CP}$ feasible and infeasible problems. The computational results on QP and QCQP problems are not provided because these problems are stored in MOSEK QPS format, which Ipopt and Knitro do not support. Note that Knitro provides three different algorithms for solving the nonlinear problems. In our comparison, we choose the direct primal-dual IPM. For Ipopt, we use MA57 library for the sparse symmetric indefinite matrix factorization, which is the same in iOptimize. Default parameter settings are used for both solvers expect that maximum iterations allowed is set at 400 .

We first focus on the computational results for solving the CP feasible problems. The last two columns of Table A.8 contain the number of iterations used by Ipopt and Knitro. Considering the average number of iterations used for solving these problems, iOptimize (12.77 iters) ranked first, Knitro (18.95 iters) ranked second, whereas Ipopt (27.84 iters) ranked last. For problems dallasm and polak3, both Knitro and Ipopt received a function evaluation error from AMPL. Ipopt failed to solve dallass to optimality in 400 iterations. Observe that Ipopt requires more than 50 iterations for three problems and Knitro requires more than 50 iterations for five problems, whereas iOptimize solves all feasible CP problems within 50 iterations.

Now we focus on the CP infeasible problems. Computational results from Ipopt and Knitro are reported in the last two columns of Table A.14. Knitro failed to solve 13 problems within 400 iterations, whereas Ipopt fails in three problems. Knitro received an AMPL function evaluation error in three problems and Ipopt received the same error in four problems. Note that both Knitro and Ipopt terminate with a near optimal status in one problem. In three problems Ipopt reported error in feasibility restoration phase. Overall, Ipopt successfully detects the infeasibility in 35 problems and Knitro in 29 problems.

6. Concluding remarks. In this paper we have extended Mehrotra-Huang potential function [26] to the context of $\mathrm{MCP}$, and shown that our potential reduction interior point method generates a maximal complementary solution with desired accuracy if a scaled Lipschitz condition is satisfied. However, the main emphasis of the analysis is not to improve the best known complexity, but to make sure the interior point method maintains the global linear polynomial time convergence properties while achieving practical performance. We have implemented an interior point solver in a software package named iOptimize that does not ignore the theory of potential function in the context of interior point methods, while adding only a small additional computational burden. Computational comparisons on feasible and infeasible test problems show that, in terms of iteration counts and the number of problems solved, iOptimize appears to perform more efficiently and robustly than a mature commercial solver MOSEK. We also find that iOptimize seems to detect infeasibility more reliably than general nonlinear solvers Ipopt and Knitro. It might be possible to further improve the computational results by implementing a better preprocessor, a non-trivial starting point, or better step size computations. However, that was not the focus of the research reported here.

At the core of the solver is the matrix factorization method, for which iOptimize uses Bunch-Parlett factorization [14]. The method used in MOSEK is not known to us. However, we point out that numerical instability may result if quasi-definite system approach is used $[11,37]$. Bunch-Parlett factorization in general is numerically stabler, but requires additional computational effort.

We also implemented a version of Gondzio's higher-order correction strategy [18] 
in iOptimize. Our implementation of this strategy is a direct extension of the approach in Mehrotra and Huang [26]. Computational results show that iOptimize using Mehrotra direction and up to two additional Gondzio's correctors for the QP problems saves only approximately $9 \%$ iterations. However, we did not see any improvement on QCQP and CP problems. The practical value of using other correction strategies is a topic of future research.

We note that the computational performance for a problem can be improved possibly with different parameter settings. For example, with parameters $\left(\theta_{l}, \theta_{u}\right)=$ $(0.99,1)$, the average iterations for solving the QCQP and CP feasible problems is reduced further by about $6 \%$. However, this number is increased by about $9 \%$ while solving the QCQP and CP infeasible problems. In fact, for antenna-iantenna_vareps, iOptimize under default setting $\left(\left(\theta_{l}, \theta_{u}\right)=(0.9,1)\right)$ needs only 47 iterations to correctly detect infeasibility whereas with parameters $\left(\theta_{l}, \theta_{u}\right)=(0.99,1)$ it will require 454 iterations. A very aggressive step to the boundary is not desirable for infeasible and general convex programming problems.

Finally, we note that a small but difficult two-variable example can be found at http://mosek.com/fileadmin/talks/func_versus_conic.pdf, (page 29). With the default setting iOptimize uses 24 iterations to solve this example to optimality whereas MOSEK with default setting uses 124 iterations.

\section{REFERENCES}

[1] AMPL: A modeling language for mathematical programming. www.ampl.com.

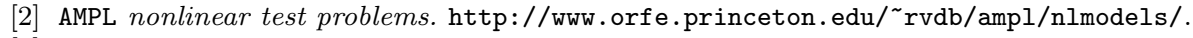

[3] COIN-OR Ipopt interior point optimizer. http://www.coin-or.org/ipopt/.

[4] Knitro: A aolver for nonlinear optimization. http://www.ziena.com/knitro.html.

[5] Mittelmann's convex QCQP test problems. http://plato.asu.edu/ftp/ampl_files/qpdata_ampl/.

[6] Mixed integer nonlinear programming (minlp) test problems. http://wiki.mcs.anl.gov/leyffer/index.php/MacMINLP.

[7] MOSEK: A high performance solftware for large-scale optimization problems. http://www.mosek.com/.

[8] Netlib. http://www.netlib.org/lp/index.html.

[9] E.D. ANDERSEN, On primal and dual infeasibility certificates in a homogeneous model for convex optimization, SIAM Journal On Optimization, 11 (2000), pp. 380-388.

[10] E.D. Andersen, C. Roos, And T. Terlaky, On implementing a primal-dual interior-point method for conic quadratic optimization, Mathematical Programming, 95 (2003), pp. 249277.

[11] E.D. Andersen AND Y. YE, A computational study of the homogeneous algorithm for largescale convex optimization, Computational Optimization and Applications, 10 (1998), pp. 243-269.

[12] - On a homogeneous algorithm for the monotone complementarity problem, Mathematical Programming, 84 (1999), pp. 375-399.

[13] M.S. Bazarra, H.D. Sherali, And C.M. Shetty, Nonlinear Programming: Theory And Algorithms, John Wiley \& Sons, New York, 2 ed., 1993.

[14] J.R. Bunch AND B.N. PARLETT, Direct methods for solving symmetric indefinite systems of linear equations, SIAM Journal on Numerical Analysis, 8 (1971), pp. 639-655.

[15] E. DOLAN AND J. MoRÉ, Benchmarking optimization software with performance profiles, Mathematical Programming, 91 (2002), pp. 201-213.

[16] I.S. DUFF, MA57-a code for the solution of sparse symmetric definite and indefinie systems, ACM Transactions on Mathematical Software, 30 (2004), pp. 118-144.

[17] D. Goldfarb and S. Mehrotra, A relaxed variant of Karmarkar's algorithm, Mathematical Programming, 40 (1988), pp. 285-315.

[18] J. Gondzio, Multiple centrality corrections in a primal-dual method for linear programming, Computational Optimization and Applications, 6 (1996), pp. 137-156.

[19] O. GüLER, Existence of interior points and interior paths in nonlinear monotone complementarity problems, Mathematics of Operations Research, 18 (1993), pp. 148-162. 
[20] O. Güler And Y. Ye, Convergence behavior of interior-point algorithms, mathematical Programming, 60 (1993), pp. 215-228.

[21] N. KARMARKAR, A new polynomial-time algorithm for linear programming, Combinatorica, 4 (1984), pp. 373-395.

[22] M. Kojima, S. Mizuno, And A. Yoshise, $A n O(\sqrt{n} L)$ iteration potential reduction algorithm for linear complementary problems, Mathematical Programming, 50 (1991), pp. 331-342.

[23] Z.-Q. Luo, J.F. Sturm, And S. Zhang, Conic convex programming and seld-dual embedding, Optimization Method and Software, 14 (2000), pp. 169-218.

[24] I. Maros and C. MÉzÁros, A repository of convex quadratic programming problems, Optimization Methods and Software, 11\&12 (1999), pp. 671-681.

[25] S. Menrotra, On the implementation of a primal-dual interior point method, SIAM Journal on Optimization, 2 (1992), pp. 575-601.

[26] S. Mehrotra And K.-L. Huang, Computational experience with a modified potential reduction algorithm for linear programming, Optimization Method and Software, 27 (2012), pp. 865891.

[27] M.D.C. Monteiro And I. Adler, An extension of Karmarkar type algorithm to a class of convex separable programming problems with global rate of convergence, Mathematical of Operations Research, 15 (1990), pp. 408-422.

[28] Y.E. Nesterov and M.J. TodD, Self-scaled barriers and interior-point methods for convex programming, Mathematics of Operations Research, 22 (1997), pp. 1-42.

[29] F. PORTA AND Y. YE, Interior-point methods for nonlinear complementarity problem, Journal of Optimization Theory and Applications, 68 (1996), pp. 617-642.

[30] F.A. POTRA AND Y. Ye, A quadratically convergent polynomial algorithm for solving entropy optimization problems, SIAM Jorunal on Optimization, 3 (1993), pp. 843-860.

[31] _ Interior point methods for nonlinear complementarity problems, Journal of Optimization Theory and Application, 88 (1996), pp. 617-647.

[32] R.T. Rockafellar, Convex Analysis, Princeton University Press, 1996.

[33] A. SkajaA, J.B. Jørgensen, And P.C. Hansen, On implementing a homogeneous interior-point algorithm for nonsymmetric conic optimization, http://www.imm.dtu.dk/English/Service/ Phonebook. aspx?lg=show common\&id=f30a529b-4c6b-4642-a67b-cca4f74d0a3a, (2011).

[34] J.F. Sturm, Using sedumi 1.02, a matlab toolbox for optimization over symmetric cones, Optimization Method and Software, 11-12 (1999), pp. 625-653.

[35] K. TANABE, Centered newton method for mathematical programming, System Modelling and Optimization, 113 (1988), pp. 197-206.

[36] M.J. TODD And Y. Ye, A centered projective algorithm for linear programming, Mathematics of Operations Research, 15 (1990), pp. 508-529.

[37] R.J. VANDERBEI, Symmetric quasi-definite matrices, SIAM Journal on Optimization, 5 (1995), pp. 100-113.

[38] X. XU, P. Hung, AND Y. YE, A simplified homogeneous and self-dual linear programming algorithm and its implementation, Annals of Operations Research, 62 (1996), pp. 151-171.

[39] Y. Ye, M.J. Todd, And S. Mizuno, An $O(\sqrt{n} L)$-iteration homogeneous and self-dual linear programming algorithm, Mathematics of Operations Research, 19 (1994), pp. 53-67.

[40] S. Zhang, A new self-dual embedding method for convex programming, Journal of Global Optimization, 29 (2004), pp. 479-496.

[41] J. ZHU, A path following alrogirhm for a class of convex programming problems, Method and Models of Operations Research, 36 (1992), pp. 359-377.

\section{Appendix. A.}




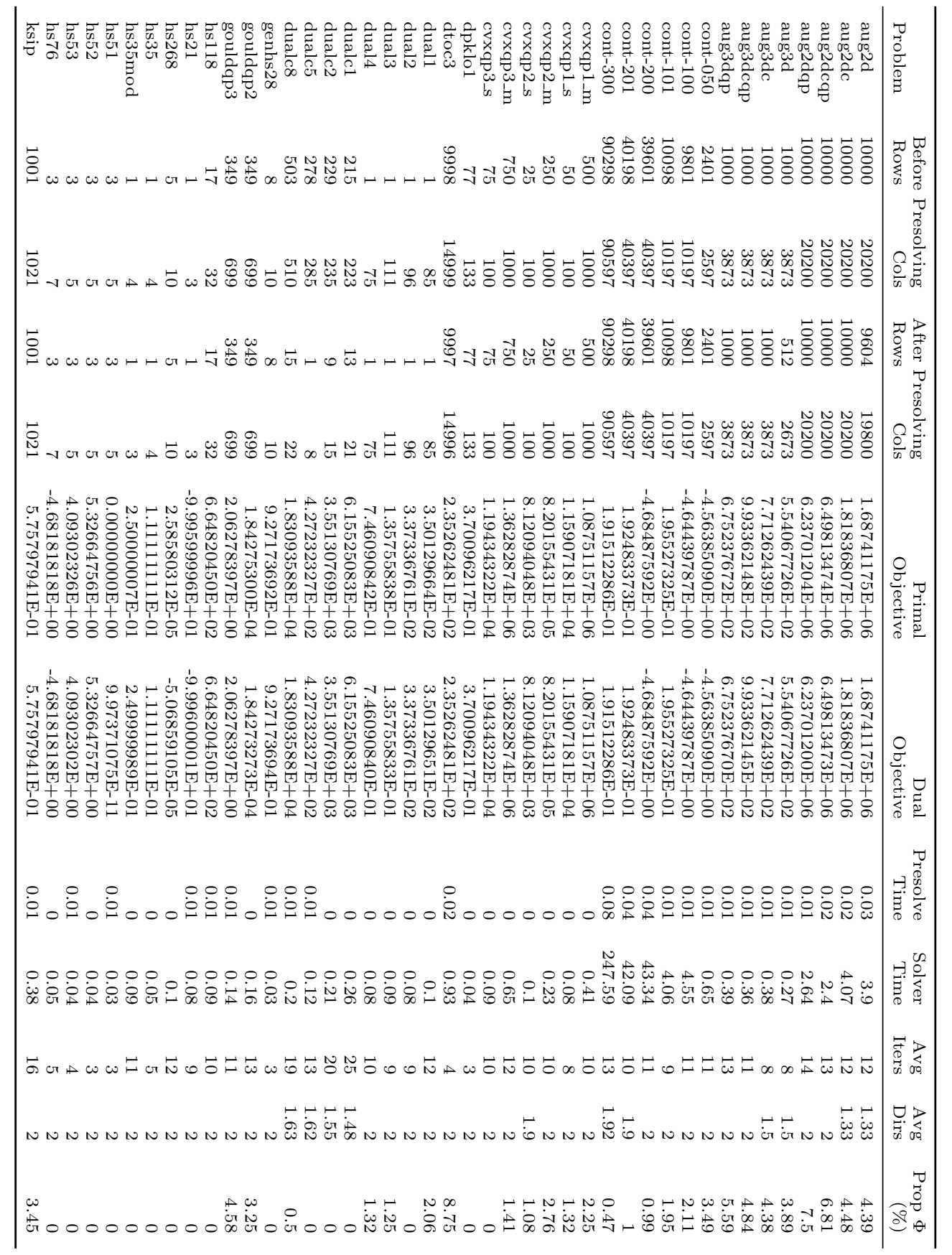




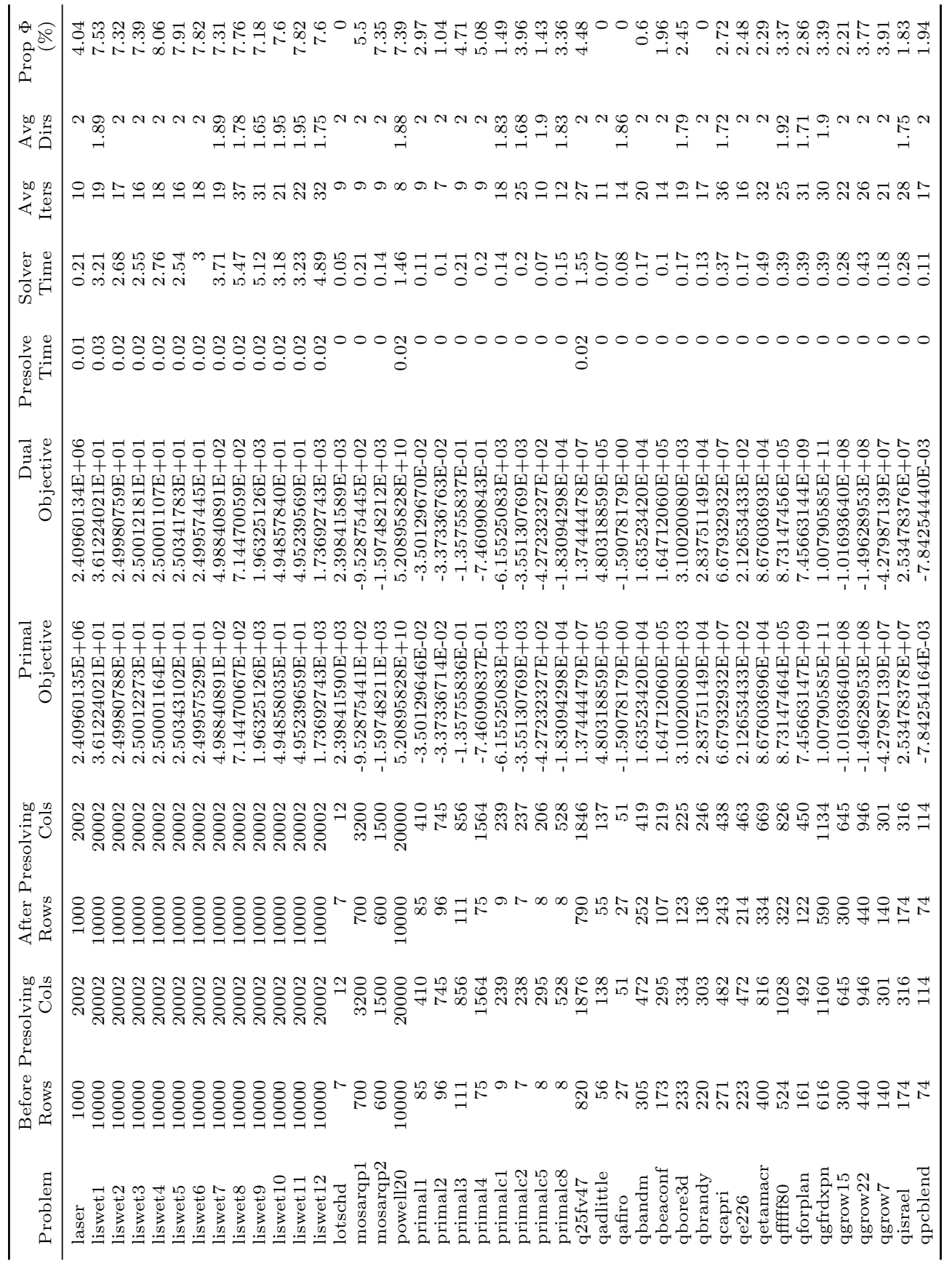




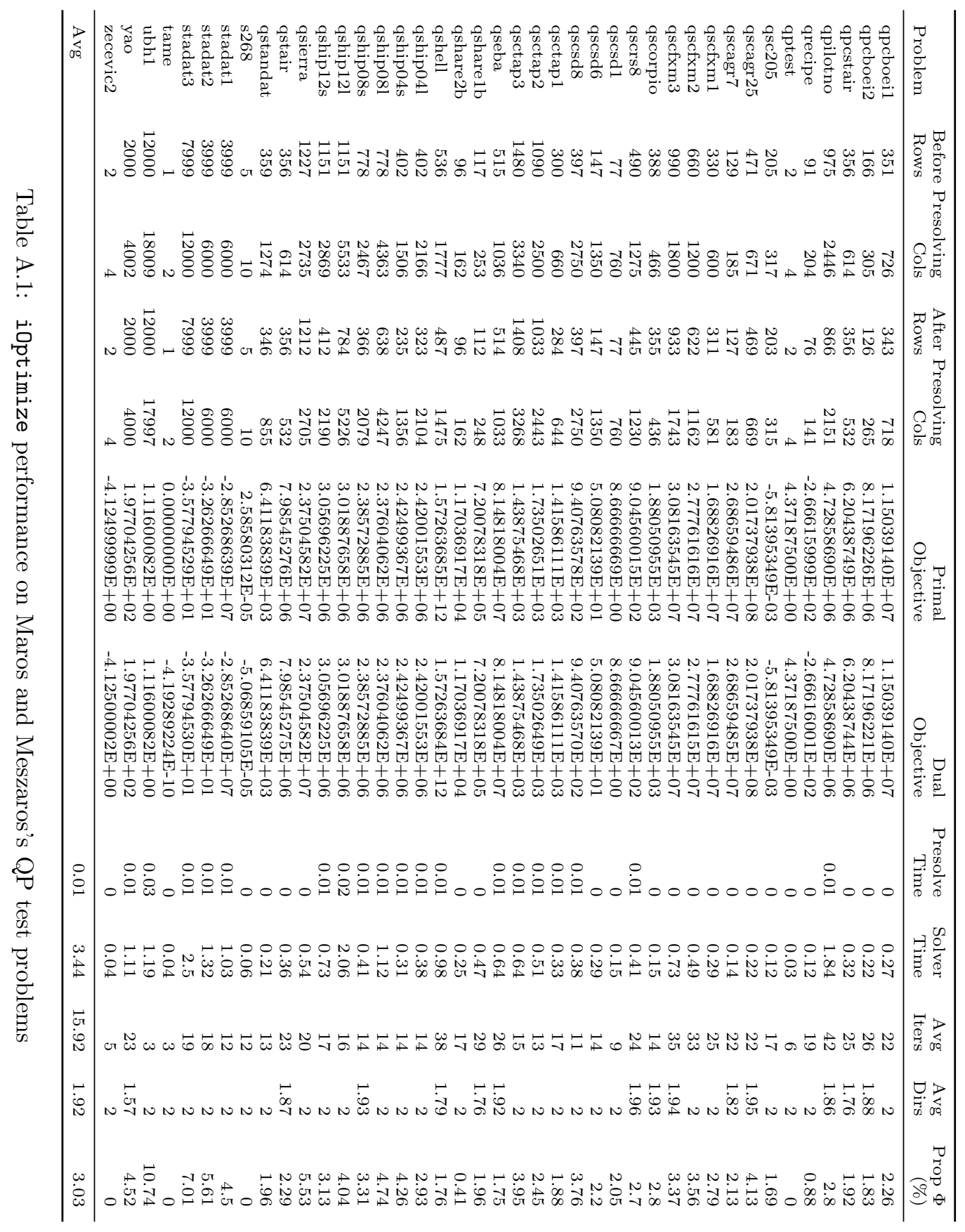




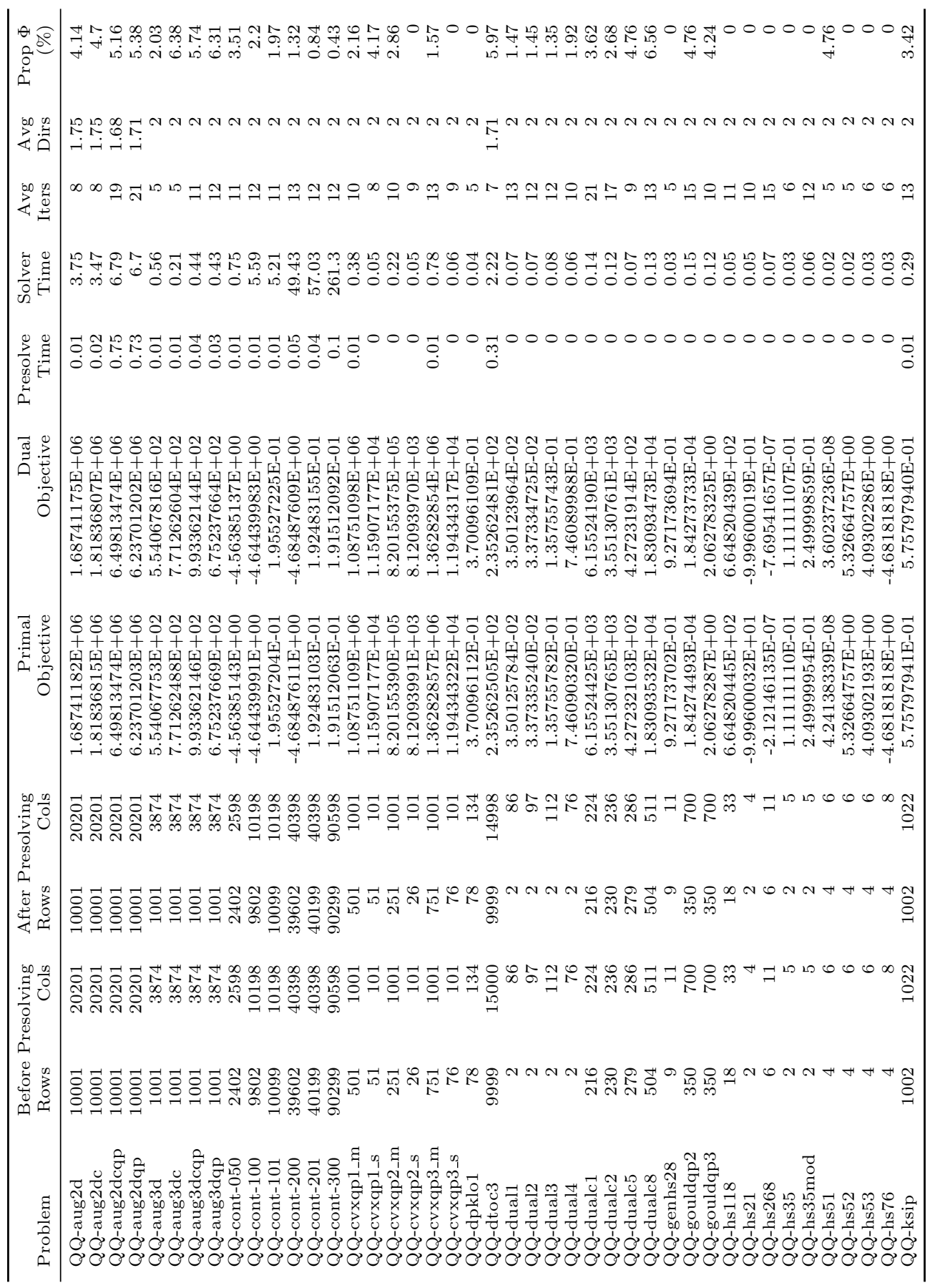




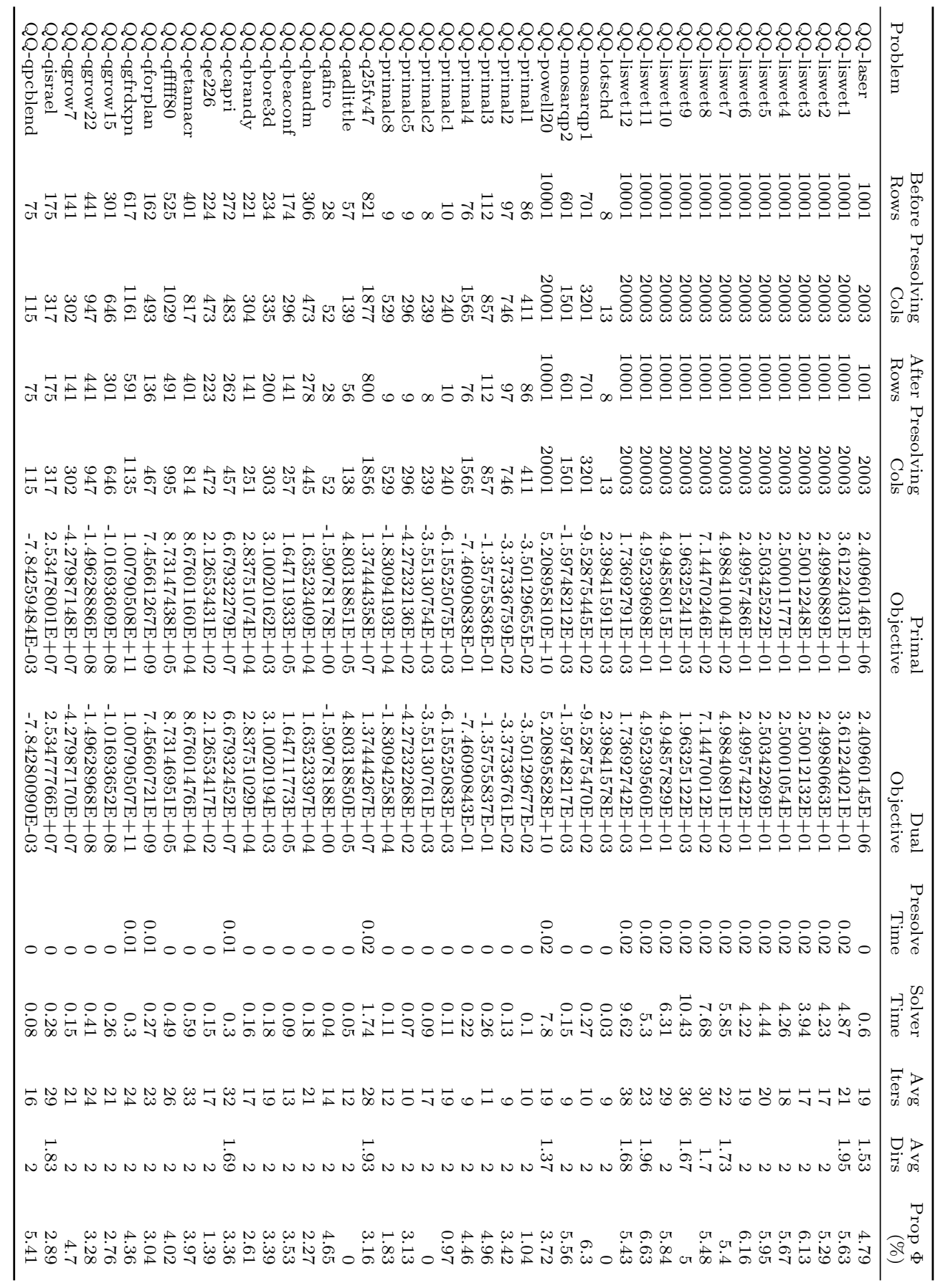




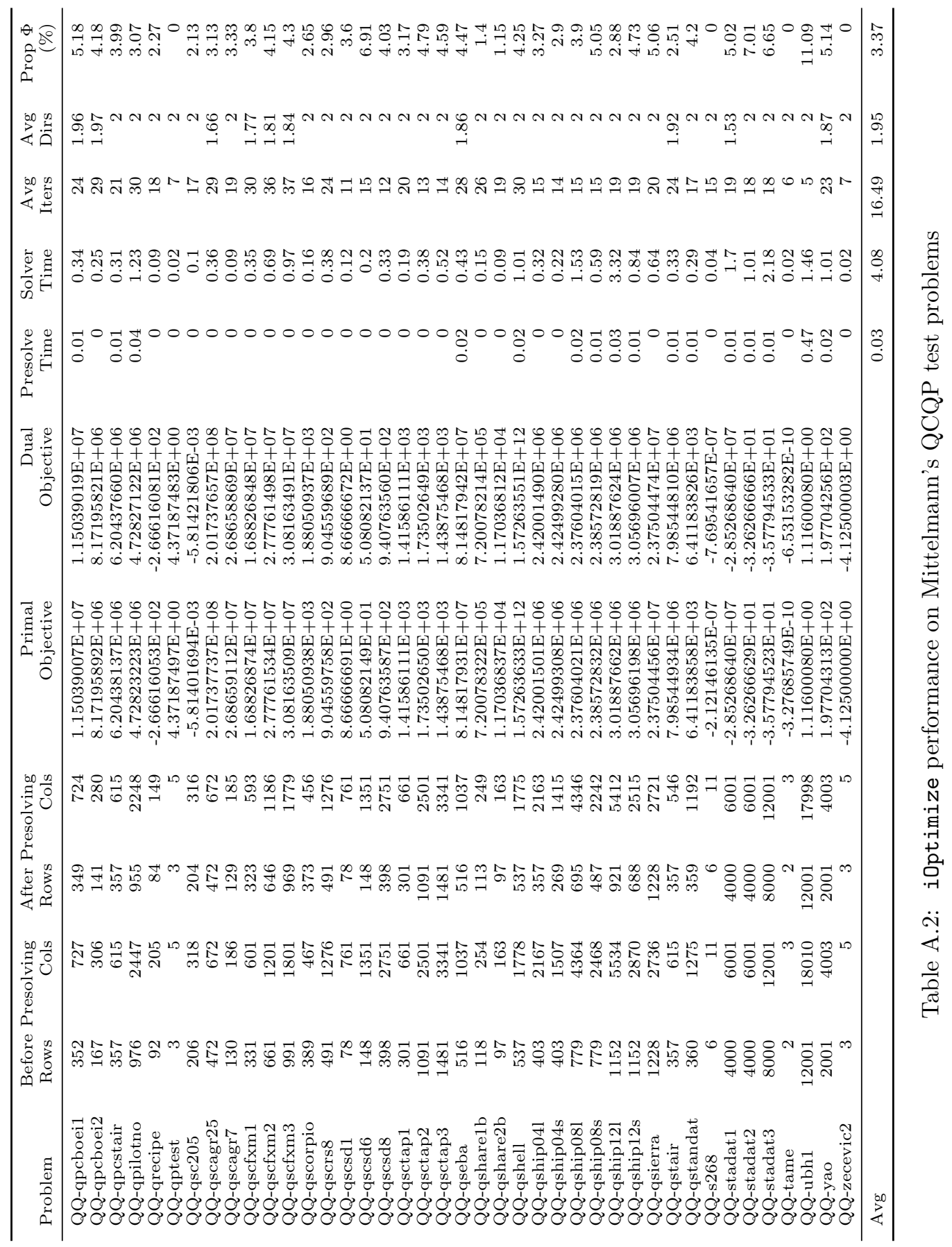




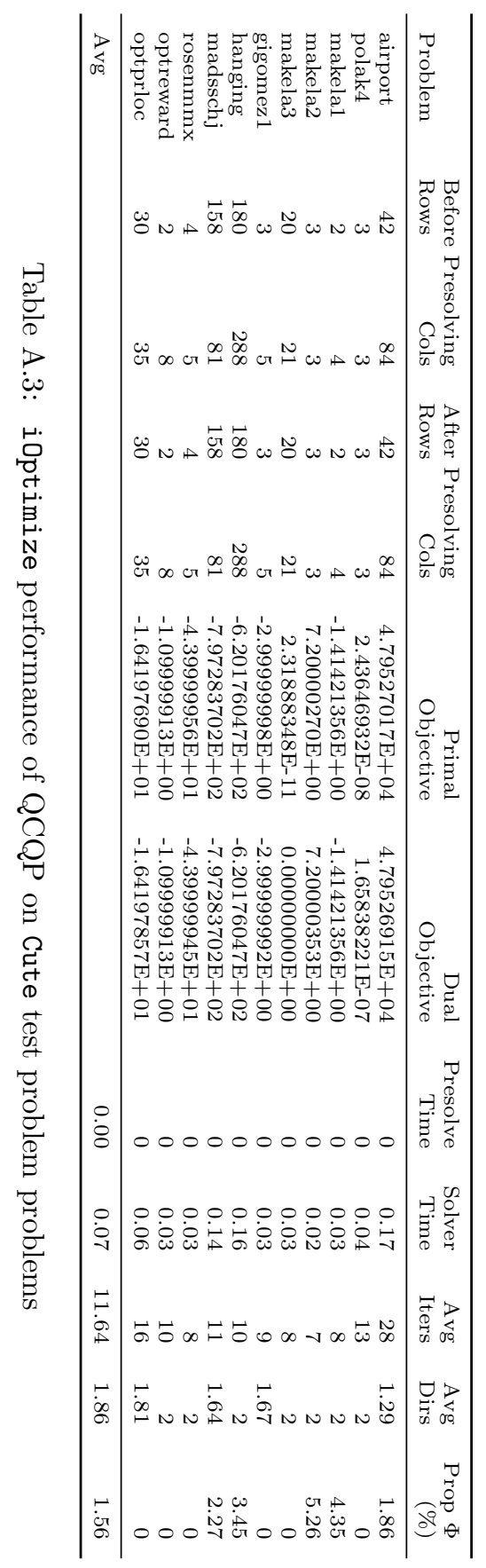




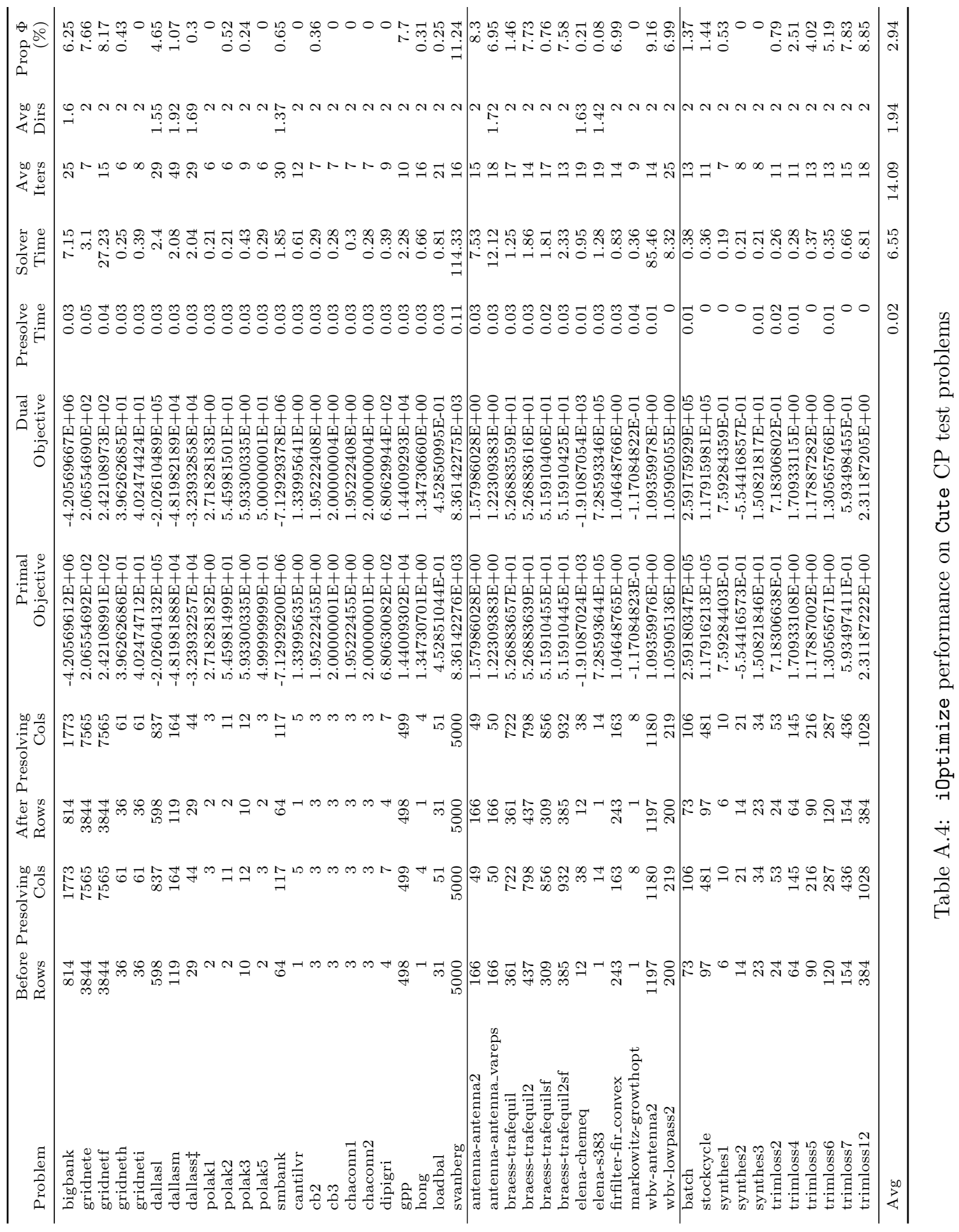




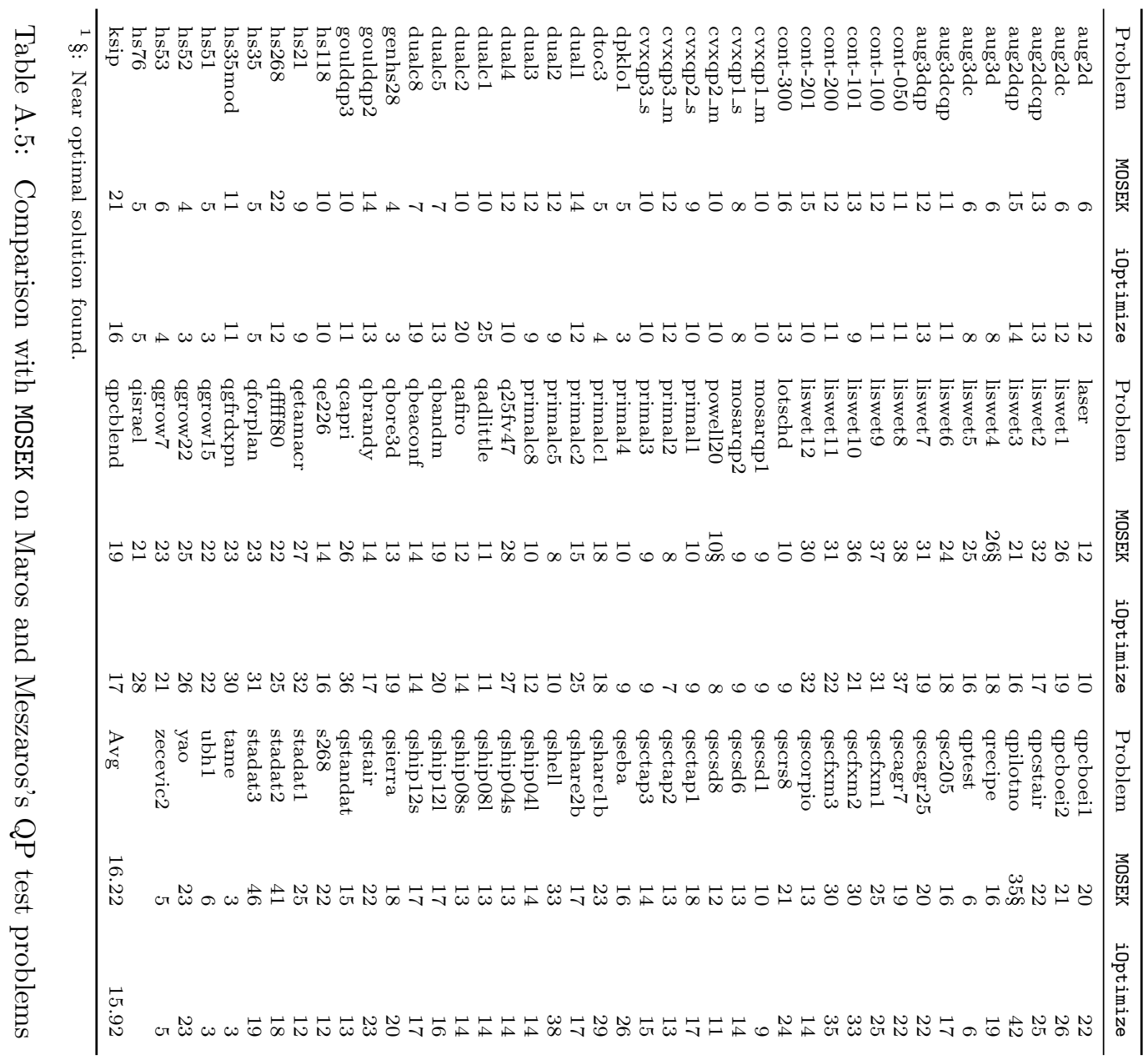




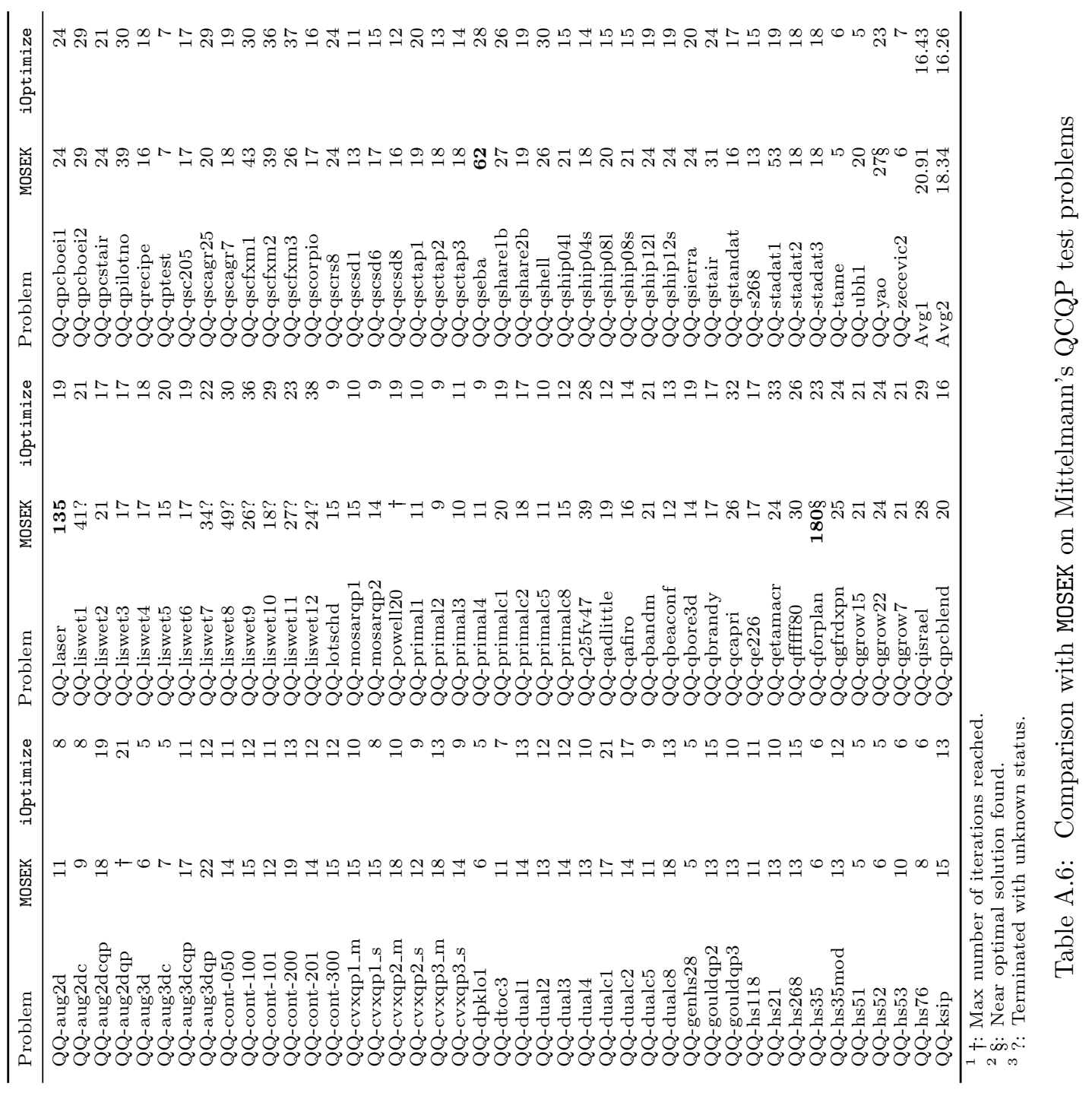




\begin{tabular}{lrr}
\hline Problem & MOSEK & iOptimize \\
\hline airport & 26 & 28 \\
polak4 & 10 & 13 \\
makela1 & 8 & 8 \\
makela2 & 7 & 7 \\
makela3 & 6 & 8 \\
gigomez1 & 8 & 9 \\
hanging & 13 & 10 \\
madsschj & 10 & 11 \\
rosenmmx & 9 & 8 \\
optreward & 10 & 10 \\
optprloc & 18 & 16 \\
\hline Avg & 11.36 & 11.64 \\
\hline
\end{tabular}

Table A.7: Comparison with MOSEK on Cute QCQP test problems 


\begin{tabular}{|c|c|c|c|c|}
\hline Problem & MOSEK & iOptimize & Ipopt & Knitro \\
\hline bigbank & 20 & 21 & 24 & 19 \\
\hline gridnete & 9 & 7 & 5 & 4 \\
\hline gridnetf & 16 & 13 & 27 & 17 \\
\hline gridneth & 8 & 6 & 6 & 3 \\
\hline gridneti & 10 & 8 & 7 & 6 \\
\hline dallasl & 74 & 33 & 249 & 245 \\
\hline dallasm & 128 & 31 & $\ddagger$ & $\ddagger$ \\
\hline dallass & $\dagger$ & 31 & $\dagger$ & 135 \\
\hline polak1 & 6 & 5 & 4 & 2 \\
\hline polak2 & 6 & 5 & 4 & 1 \\
\hline polak3 & 10 & 8 & $\ddagger$ & $\ddagger$ \\
\hline polak5 & 6 & 5 & 24 & 12 \\
\hline smbank & 14 & 27 & 12 & 11 \\
\hline cantilvr & 12 & 12 & 5 & 3 \\
\hline $\mathrm{cb} 2$ & 8 & 8 & 4 & 3 \\
\hline cb3 & 7 & 7 & 4 & 4 \\
\hline chaconn 1 & 8 & 8 & 4 & 3 \\
\hline chaconn 2 & 7 & 7 & 4 & 4 \\
\hline dipigri & 14 & 10 & 6 & 2 \\
\hline gpp & 12 & 10 & 22 & 11 \\
\hline hong & 13 & 15 & 11 & 7 \\
\hline loadbal & 22 & 21 & 13 & 7 \\
\hline svanberg & 14 & 14 & 30 & 16 \\
\hline antenna \antenna2 & $21 \S$ & 16 & 178 & 58 \\
\hline antenna \antenna_vareps & 19 & 18 & 106 & 61 \\
\hline braess $\backslash$ trafequil & 15 & 16 & 18 & 22 \\
\hline braess $\backslash$ trafequil2 & 11 & 13 & 26 & 13 \\
\hline braess \trafequilsf & 14 & 15 & 18 & 24 \\
\hline braess $\backslash$ trafequil2sf & 12 & 12 & 25 & 12 \\
\hline elena $\backslash$ chemeq & 23 & 20 & 37 & 16 \\
\hline elena $\backslash \mathrm{s} 383$ & 85 & 21 & 18 & 8 \\
\hline firfilter $\backslash$ fir_convex & $33 \S$ & 13 & 27 & 11 \\
\hline markowitz \growthopt & 8 & 7 & 8 & 7 \\
\hline wbv \antenna2 & 15 & 13 & 31 & 16 \\
\hline wbv $\backslash$ lowpass 2 & $30 \S$ & 22 & 31 & 66 \\
\hline batch & 13 & 12 & 13 & 16 \\
\hline stockcycle & 11 & 10 & 23 & 12 \\
\hline synthes1 & 6 & 6 & 6 & 6 \\
\hline synthes 2 & 8 & 8 & 10 & 7 \\
\hline synthes 3 & 8 & 8 & 9 & 8 \\
\hline trimloss 2 & 10 & 10 & 13 & 7 \\
\hline trimloss 4 & 11 & 12 & 16 & 8 \\
\hline trimloss 5 & 13 & 13 & 20 & 10 \\
\hline trimloss 6 & 12 & 12 & 23 & 11 \\
\hline trimloss 7 & 13 & 13 & 35 & 14 \\
\hline trimloss 12 & 19 & 17 & 41 & 22 \\
\hline Avg1 & 18.46 & 14.00 & - & - \\
\hline Avg2 & 13.07 & 12.69 & - & - \\
\hline
\end{tabular}

Table A.8: Comparison with MOSEK, Ipopt, and Knitro on CP test problems 


\begin{tabular}{|c|c|c|c|c|c|}
\hline Problem & $\begin{array}{c}\text { Presolve } \\
\text { Time }\end{array}$ & $\begin{array}{c}\text { Solver } \\
\text { Time }\end{array}$ & $\begin{array}{l}\text { Avg } \\
\text { Iters }\end{array}$ & $\begin{array}{l}\text { Avg } \\
\text { Dirs }\end{array}$ & $\begin{array}{r}\text { Prop } \Phi \\
(\%)\end{array}$ \\
\hline iQQ-aug2d & 0.01 & 7.14 & 16 & 1.69 & 4.78 \\
\hline iQQ-aug2dc & 0.01 & 6.8 & 16 & 1.69 & 4.34 \\
\hline iQQ-aug2dcqp & 0.78 & 13.44 & 29 & 1.34 & 4.5 \\
\hline iQQ-aug2dqp & 0.73 & 13.4 & 29 & 1.34 & 4.44 \\
\hline iQQ-aug3d & 0 & 4.72 & 35 & 1.09 & 2.43 \\
\hline iQQ-aug3dc & 0 & 1.06 & 16 & 1.5 & 5.13 \\
\hline iQQ-aug3dcqp & 0.04 & 2.59 & 33 & 1.09 & 4.1 \\
\hline iQQ-aug3dqp & 0.03 & 2.68 & 37 & 1.05 & 4.4 \\
\hline iQQ-cont-050 & 0 & 0.76 & 9 & 2 & 4.72 \\
\hline iQQ-cont-100 & 0.01 & 6.7 & 14 & 1.93 & 2.17 \\
\hline iQQ-cont-101 & 0 & 3.33 & 9 & 2 & 2.35 \\
\hline iQQ-cont-200 & 0.03 & 47.74 & 14 & 2 & 1.71 \\
\hline iQQ-cont-201 & 0.03 & 26.08 & 9 & 2 & 2.09 \\
\hline iQQ-cont-300 & 0.05 & 104.79 & 9 & 2 & 1.57 \\
\hline iQQ-cvxqp1_m & 0 & 2.19 & 26 & 1.38 & 1.38 \\
\hline iQQ-cvxqp1_s & 0 & 2.24 & 35 & 1.2 & 0.45 \\
\hline iQQ-cvxqp2_m & 0 & 2.14 & 29 & 1.34 & 0.9 \\
\hline iQQ-cvxqp2_s & 0 & 2.41 & 37 & 1.19 & 0.92 \\
\hline iQQ-cvxqp3_m & 0 & 2.85 & 30 & 1.23 & 1.8 \\
\hline iQQ-cvxqp3_s & 0 & 1.22 & 35 & 1.17 & 0.98 \\
\hline iQQ-dpklo1 & 0 & 0.36 & 13 & 2 & 0.83 \\
\hline iQQ-dtoc3 & 0.32 & 4.21 & 15 & 1.87 & 5.9 \\
\hline iQQ-dual1 & 0 & 1.14 & 31 & 1.32 & 0.88 \\
\hline iQQ-dual2 & 0 & 0.52 & 16 & 1.69 & 0 \\
\hline iQQ-dual3 & 0 & 0.63 & 18 & 1.67 & 1.61 \\
\hline iQQ-dual4 & 0 & 1.23 & 33 & 1.33 & 0 \\
\hline iQQ-dualc1 & 0 & 1.06 & 35 & 1.66 & 0 \\
\hline iQQ-dualc2 & 0 & 1.19 & 35 & 1.57 & 0.59 \\
\hline iQQ-dualc5 & 0 & 1.65 & 44 & 1.32 & 1.04 \\
\hline iQQ-dualc8 & 0 & 1.06 & 34 & 1.68 & 1.9 \\
\hline iQQ-genhs 28 & 0 & 0.37 & 13 & 2 & 0 \\
\hline iQQ-gouldqp2 & 0 & 0.27 & 10 & 2 & 0 \\
\hline iQQ-gouldqp3 & 0 & 0.56 & 16 & 1.63 & 1.82 \\
\hline iQQ-hs118 & 0 & 0.27 & 11 & 2 & 0 \\
\hline iQQ-hs 21 & 0.02 & 1.2 & 35 & 1.11 & 0.08 \\
\hline iQQ-hs268 & 0 & 1.52 & 45 & 1.11 & 0 \\
\hline iQQ-hs35 & 0 & 0.52 & 16 & 1.75 & 0 \\
\hline iQQ-hs35mod & 0 & 0.54 & 16 & 1.75 & 0.19 \\
\hline iQQ-hs51 & 0 & 1.07 & 27 & 1.19 & 0 \\
\hline iQQ-hs52 & 0 & 0.73 & 26 & 1.31 & 0 \\
\hline iQQ-hs53 & 0 & 1.41 & 39 & 1.13 & 0 \\
\hline iQQ-hs76 & 0 & 0.36 & 13 & 2 & 0 \\
\hline iQQ-ksip & 0 & 1.73 & 42 & 1.05 & 3.3 \\
\hline
\end{tabular}




\begin{tabular}{lrrrrr}
\hline & $\begin{array}{r}\text { Presolve } \\
\text { Time }\end{array}$ & $\begin{array}{r}\text { Solver } \\
\text { Time }\end{array}$ & $\begin{array}{r}\text { Avg } \\
\text { Iters }\end{array}$ & $\begin{array}{r}\text { Avg } \\
\text { Dirs }\end{array}$ & $\begin{array}{r}\text { Prop } \Phi \\
\text { Problem }\end{array}$ \\
\hline iQQ-laser & 0 & 0.4 & 13 & 2 & 5.22 \\
iQQ-liswet1 & 0.01 & 16.43 & 42 & 1.14 & 4.19 \\
iQQ-liswet2 & 0.02 & 17.23 & 43 & 1.12 & 4.9 \\
iQQ-liswet3 & 0.01 & 15 & 38 & 1.16 & 5.15 \\
iQQ-liswet4 & 0.01 & 15.72 & 40 & 1.13 & 5.16 \\
iQQ-liswet5 & 0.01 & 18.09 & 47 & 1.17 & 4.49 \\
iQQ-liswet6 & 0.02 & 16.79 & 43 & 1.14 & 4.64 \\
iQQ-liswet7 & 0.01 & 15.81 & 40 & 1.13 & 5.12 \\
iQQ-liswet8 & 0.01 & 14.03 & 33 & 1.15 & 4.37 \\
iQQ-liswet9 & 0.01 & 15.69 & 37 & 1.14 & 4.69 \\
iQQ-liswet10 & 0.02 & 16.5 & 42 & 1.14 & 4.97 \\
iQQ-liswet11 & 0.01 & 16.33 & 42 & 1.14 & 4.9 \\
iQQ-liswet12 & 0.01 & 15.73 & 38 & 1.13 & 4.62 \\
iQQ-lotschd & 0 & 0.3 & 12 & 2 & 0 \\
iQQ-mosarqp1 & 0 & 2.04 & 33 & 1.09 & 4.55 \\
iQQ-mosarqp2 & 0 & 1.41 & 38 & 1.08 & 3.57 \\
iQQ-powell20 & 0.02 & 6.5 & 16 & 1.63 & 3.5 \\
iQQ-primal1 & 0.01 & 1.28 & 43 & 1.09 & 1.41 \\
iQQ-primal2 & 0 & 1.31 & 37 & 1.14 & 2.47 \\
iQQ-primal3 & 0 & 1.58 & 41 & 1.1 & 2.24 \\
iQQ-primal4 & 0.01 & 1.56 & 36 & 1.17 & 4.44 \\
iQQ-primalc1 & 0 & 1.37 & 42 & 1.26 & 1.03 \\
iQQ-primalc2 & 0 & 1.32 & 36 & 1.33 & 1.52 \\
iQQ-primalc5 & 0 & 0.97 & 23 & 1.39 & 0.93 \\
iQQ-primalc8 & 0 & 1.1 & 32 & 1.28 & 1.01 \\
iQQ-q25fv47 & 0.01 & 1.4 & 15 & 2 & 6.42 \\
iQQ-qadlittle & 0 & 0.34 & 14 & 2 & 0.59 \\
iQQ-qafiro & 0 & 1.37 & 39 & 1.15 & 0.07 \\
iQQ-qbandm & 0 & 0.34 & 14 & 1.86 & 3.02 \\
iQQ-qbeaconf & 0 & 0.41 & 14 & 1.93 & 0.5 \\
iQQ-qbore3d & 0 & 0.51 & 18 & 1.89 & 0 \\
iQQ-qbrandy & 0 & 0.48 & 16 & 1.88 & 0.41 \\
iQQ-qcapri & 0 & 0.62 & 22 & 1.91 & 1.63 \\
iQQ-qe226 & 0 & 1.41 & 36 & 1.22 & 1.85 \\
iQQ-qetamacr & 0 & 1.09 & 34 & 1.21 & 3.17 \\
iQQ-qfffff80 & 0 & 0.57 & 20 & 1.8 & 3.57 \\
iQQ-qforplan & 0.01 & 0.25 & 11 & 2 & 1.63 \\
iQQ-qgfrdxpn & 0.01 & 0.47 & 19 & 2 & 2.42 \\
iQQ-qgrow15 & 0 & 0.44 & 16 & 1.94 & 1.15 \\
iQQ-qgrow22 & 0.01 & 0.54 & 18 & 1.89 & 0 \\
iQQ-qgrow7 & 0 & 0.56 & 18 & 1.89 & 0.54 \\
iQQ-qisrael & 0 & 0.47 & 19 & 2 & 1.3 \\
iQQ-qpcblend & 0 & 1.5 & 38 & 1.16 & 0.27 \\
\hline & & & & & \\
\hline
\end{tabular}




\begin{tabular}{|c|c|c|c|c|c|}
\hline Problem & $\begin{array}{c}\text { Presolve } \\
\text { Time }\end{array}$ & $\begin{array}{r}\text { Solver } \\
\text { Time }\end{array}$ & $\begin{array}{l}\text { Avg } \\
\text { Iters }\end{array}$ & $\begin{array}{l}\text { Avg } \\
\text { Dirs }\end{array}$ & $\begin{array}{r}\text { Prop } \Phi \\
(\%)\end{array}$ \\
\hline iQQ-qpcboei1 & 0.01 & 0.31 & 12 & 2 & 6.06 \\
\hline iQQ-qpcboei2 & 0 & 0.44 & 17 & 1.94 & 4.65 \\
\hline iQQ-qpcstair & 0.01 & 0.64 & 20 & 1.75 & 1.27 \\
\hline iQQ-qpilotno & 0.04 & 0.65 & 16 & 2 & 3.84 \\
\hline iQQ-qrecipe & 0 & 0.75 & 20 & 1.6 & 0.13 \\
\hline iQQ-qptest & 0 & 0.56 & 16 & 1.81 & 0.18 \\
\hline iQQ-qsc205 & 0 & 1.51 & 41 & 1.24 & 1.35 \\
\hline iQQ-qscagr25 & 0 & 0.32 & 14 & 2 & 0.32 \\
\hline iQQ-qscagr7 & 0 & 0.33 & 14 & 2 & 1.22 \\
\hline iQQ-qscfxm1 & 0 & 0.4 & 15 & 1.93 & 1.79 \\
\hline iQQ-qscfxm2 & 0 & 0.42 & 16 & 1.94 & 2.44 \\
\hline iQQ-qscfxm3 & 0 & 0.54 & 16 & 1.94 & 4.71 \\
\hline iQQ-qscorpio & 0 & 0.95 & 25 & 1.24 & 1.91 \\
\hline iQQ-qscrs8 & 0 & 0.63 & 22 & 1.73 & 6.45 \\
\hline iQQ-qscsd1 & 0 & 0.84 & 20 & 1.6 & 1.31 \\
\hline iQQ-qscsd6 & 0 & 0.89 & 22 & 1.64 & 2.07 \\
\hline iQQ-qscsd8 & 0 & 2.02 & 37 & 1.11 & 5.11 \\
\hline iQQ-qsctap1 & 0 & 1.07 & 36 & 1.19 & 1.14 \\
\hline iQQ-qsctap2 & 0 & 2.26 & 40 & 1.13 & 3.58 \\
\hline iQQ-qsctap3 & 0 & 2.26 & 32 & 1.22 & 3.65 \\
\hline iQQ-qseba & 0.02 & 0.57 & 20 & 1.85 & 5.36 \\
\hline iQQ-qshare1b & 0 & 0.44 & 17 & 1.94 & 2.33 \\
\hline iQQ-qshare $2 b$ & 0 & 0.47 & 13 & 1.69 & 2.13 \\
\hline iQQ-qshell & 0.02 & 0.82 & 17 & 2 & 6.4 \\
\hline iQQ-qship04l & 0 & 0.31 & 11 & 2 & 0 \\
\hline iQQ-qship04s & 0.01 & 0.25 & 11 & 2 & 6.88 \\
\hline iQQ-qship08l & 0.01 & 1.63 & 16 & 1.94 & 4.56 \\
\hline iQQ-qship08s & 0 & 0.72 & 17 & 1.88 & 3.73 \\
\hline iQQ-qship12l & 0.01 & 2.96 & 17 & 1.71 & 4.74 \\
\hline iQQ-qship12s & 0.01 & 0.99 & 18 & 1.83 & 2.98 \\
\hline iQQ-qsierra & 0 & 0.64 & 17 & 2 & 5 \\
\hline iQQ-qstair & 0 & 0.39 & 17 & 2 & 1.05 \\
\hline iQQ-qstandat & 0.01 & 1.37 & 39 & 1.18 & 2.05 \\
\hline iQQ-s268 & 0 & 1.56 & 45 & 1.11 & 0.06 \\
\hline iQQ-stadat1 & 0.01 & 3.13 & 26 & 1.23 & 4.19 \\
\hline iQQ-stadat2 & 0 & 4.39 & 35 & 1.14 & 4.42 \\
\hline iQQ-stadat3 & 0.01 & 6.18 & 25 & 1.2 & 4.25 \\
\hline iQQ-tame & 0 & 0.23 & 10 & 2 & 0 \\
\hline iQQ-ubh1 & 0.46 & 5.73 & 26 & 1.96 & 6.21 \\
\hline iQQ-yao & 0.01 & 3.17 & 38 & 1.05 & 4.85 \\
\hline iQQ-zecevic2 & 0 & 0.23 & 9 & 2 & 0 \\
\hline Avg & 0.02 & 4.40 & 25.30 & 1.56 & 2.48 \\
\hline
\end{tabular}

Table A.9: iOptimize performance on Maros and Meszaros's QCQP infeasible test problems

\begin{tabular}{lrrrrr}
\hline Problem & $\begin{array}{r}\text { Presolve } \\
\text { Time }\end{array}$ & $\begin{array}{r}\text { Solver } \\
\text { Time }\end{array}$ & $\begin{array}{r}\text { Avg } \\
\text { Iters }\end{array}$ & $\begin{array}{r}\text { Avg } \\
\text { Dirs }\end{array}$ & $\begin{array}{r}\text { Prop } \Phi \\
(\%)\end{array}$ \\
\hline iairport & 0.01 & 0.6 & 19 & 1.63 & 0.67 \\
ipolak4 & 0 & 0.81 & 25 & 1.28 & 0.12 \\
imakela1 & 0 & 1.11 & 32 & 1.13 & 0 \\
imakela2 & 0 & 1.13 & 32 & 1.13 & 0 \\
imakela3 & 0 & 0.32 & 14 & 2 & 0 \\
igigomez1 & 0 & 1.02 & 26 & 1.19 & 0 \\
ihanging & 0 & 1.17 & 30 & 1.13 & 4.27 \\
imadsschj & 0.06 & 0.53 & 17 & 1.59 & 0.76 \\
irosenmmx & 0 & 1 & 35 & 1.14 & 0 \\
ioptreward & 0 & 0.64 & 20 & 1.55 & 0 \\
ioptprloc & 0 & 0.92 & 21 & 1.52 & 0.11 \\
\hline Avg & 0.01 & 0.84 & 24.64 & 1.39 & 0.54 \\
\hline
\end{tabular}

Table A.10: iOptimize performance on Cute QCQP infeasible test problems 


\begin{tabular}{|c|c|c|c|c|c|}
\hline Problem & $\begin{array}{r}\text { Presolve } \\
\text { Time }\end{array}$ & $\begin{array}{c}\text { Solver } \\
\text { Time }\end{array}$ & $\begin{array}{l}\text { Avg } \\
\text { Iters }\end{array}$ & $\begin{array}{l}\text { Avg } \\
\text { Dirs }\end{array}$ & $\begin{array}{r}\text { Prop } \Phi \\
(\%)\end{array}$ \\
\hline ibigbank & 0 & 17.12 & 27 & 1.33 & 6.06 \\
\hline igridnete & 0 & 81.51 & 14 & 1.71 & 6.79 \\
\hline igridnetf & 0 & 377.58 & 36 & 1.08 & 5.67 \\
\hline igridneth & 0.02 & 0.78 & 23 & 1.39 & 0.26 \\
\hline igridneti & 0 & 1.23 & 40 & 1.07 & 1.15 \\
\hline \multicolumn{6}{|l|}{ idallasl } \\
\hline idallasm & 0.01 & 1.96 & 47 & 1.4 & 2.26 \\
\hline idallass & 0 & 1.58 & 45 & 1.22 & 0.51 \\
\hline ipolak1 & 0 & 0.41 & 16 & 1.81 & 0 \\
\hline ipolak2 & 0 & 0.32 & 13 & 2 & 0 \\
\hline ipolak3 & 0 & 0.77 & 27 & 1.3 & 0.52 \\
\hline ipolak5 & 0 & 0.41 & 13 & 2 & 0.25 \\
\hline ismbank & 0 & 1.13 & 31 & 1.13 & 1.7 \\
\hline icantilvr & 0 & 0.29 & 12 & 2 & 0.35 \\
\hline icb2 & 0 & 0.94 & 32 & 1.19 & 0 \\
\hline icb3 & 0.01 & 1.25 & 31 & 1.16 & 0.24 \\
\hline ichaconn1 & 0 & 0.95 & 32 & 1.19 & 0 \\
\hline ichaconn 2 & 0 & 1.26 & 31 & 1.16 & 0.08 \\
\hline idipigri & 0 & 1.29 & 32 & 1.19 & 0.08 \\
\hline igpp & 0 & 34.24 & 38 & 1.11 & 5.34 \\
\hline ihong & 0 & 0.92 & 22 & 1.45 & 0.11 \\
\hline iloadbal & 0 & 0.37 & 14 & 2 & 0.27 \\
\hline isvanberg & 0.08 & 632.99 & 27 & 1.22 & 6.65 \\
\hline antenna-iantenna2 & 0 & 68.79 & 186 & 1.65 & 13.31 \\
\hline antenna-iantenna_vareps & 0 & 48.02 & 47 & 1.13 & 5.83 \\
\hline braess-itrafequil & 0 & 4.77 & 38 & 1.37 & 3.1 \\
\hline braess-itrafequil2 & 0 & 1.87 & 9 & 2 & 7.34 \\
\hline braess-itrafequilsf & 0 & 9.23 & 46 & 1.26 & 2.71 \\
\hline braess-itrafequil2sf & 0 & 2.56 & 9 & 2 & 7.16 \\
\hline elena-ichemeq & 0 & 0.06 & 14 & 2 & 4.92 \\
\hline elena-is383 & 0 & 0.06 & 14 & 1.93 & 1.64 \\
\hline firfilter-ifir_convex & 0 & 0.75 & 13 & 1.92 & 6.03 \\
\hline markowitz-igrowthopt & 0 & 0.08 & 16 & 1.69 & 0 \\
\hline wbv-iantenna 2 & 0.06 & 119.88 & 17 & 2 & 7.97 \\
\hline wbv-ilowpass 2 & 0 & 4.54 & 13 & 1.92 & 7.35 \\
\hline ibatch & 0 & 0.15 & 22 & 1.36 & 3.47 \\
\hline istockcycle & 0 & 0.88 & 35 & 1.14 & 6.29 \\
\hline isynthes1 & 0 & 0.09 & 25 & 1.36 & 0 \\
\hline isynthes 2 & 0 & 0.09 & 23 & 1.35 & 1.15 \\
\hline isynthes 3 & 0 & 0.18 & 40 & 1.1 & 3.91 \\
\hline itrimloss2 & 0 & 0.1 & 15 & 1.93 & 0 \\
\hline itrimloss 4 & 0 & 0.15 & 16 & 1.94 & 7.43 \\
\hline itrimloss5 & 0 & 0.22 & 15 & 2 & 6.07 \\
\hline itrimloss6 & 0 & 0.37 & 15 & 2 & 7.32 \\
\hline itrimloss7 & 0 & 0.84 & 16 & 2 & 6.58 \\
\hline itrimloss 12 & 0.01 & 7.79 & 16 & 2 & 7.41 \\
\hline Avg & 0.00 & 31.79 & 28.07 & 1.56 & 3.45 \\
\hline
\end{tabular}

${ }^{1} \ddagger$ : Function evaluate error from AMPL.

Table A.11: iOptimize performance on Cute CP infeasible test problems 


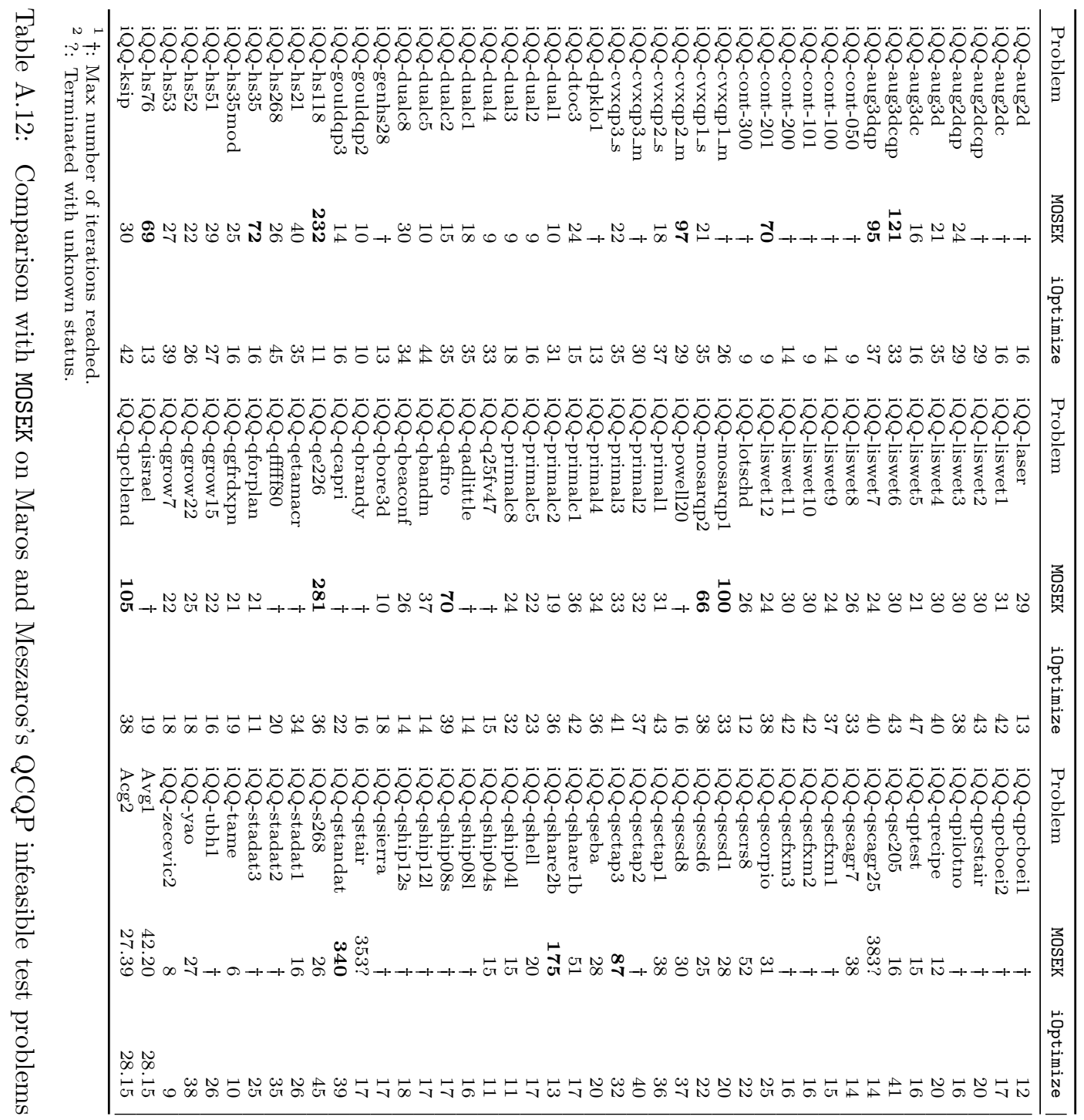




\begin{tabular}{|c|c|c|}
\hline Problem & MOSEK & iOptimize \\
\hline iairport & 10 & 19 \\
\hline ipolak4 & $\dagger$ & 25 \\
\hline imakela1 & 28 & 32 \\
\hline imakela2 & 29 & 32 \\
\hline imakela3 & 8 & 14 \\
\hline igigomez1 & 26 & 26 \\
\hline ihanging & 32 & 30 \\
\hline imadsschj & 24 & 17 \\
\hline irosenmmx & 27 & 35 \\
\hline ioptreward & 11 & 20 \\
\hline ioptprloc & 8 & 21 \\
\hline Avg & 20.30 & 24.60 \\
\hline
\end{tabular}

Table A.13: Comparison with MOSEK on Cute QCQP infeasible test problems 


\begin{tabular}{|c|c|c|c|c|}
\hline Problem & MOSEK & iOptimize & Ipopt & Knitro \\
\hline ibigbank & 196 & 27 & 48 & 42 \\
\hline igridnete & 134 & 14 & 21 & $\dagger$ \\
\hline igridnetf & $t$ & 36 & 42 & $\dagger$ \\
\hline igridneth & 202 & 23 & 25 & 18 \\
\hline igridneti & + & 40 & 40 & $133 \S$ \\
\hline idallasl & $\ddagger$ & $\ddagger$ & $50 \S$ & $\ddagger$ \\
\hline idallasm & $\ddagger$ & 47 & $\ddagger$ & 0 \\
\hline idallass & $\ddagger$ & 45 & $\ddagger$ & 0 \\
\hline ipolak1 & 50 & 16 & $\ddagger$ & $\ddagger$ \\
\hline ipolak2 & 7 & 13 & $\ddagger$ & $\ddagger$ \\
\hline ipolak3 & 178 & 27 & $\diamond$ & $\dagger$ \\
\hline ipolak5 & 7 & 13 & 37 & $\dagger$ \\
\hline ismbank & 165 & 31 & 34 & 37 \\
\hline icantilvr & 0 & 12 & 25 & 0 \\
\hline icb2 & 196 & 32 & $\diamond$ & 18 \\
\hline icb3 & 196 & 31 & $\dagger$ & 8 \\
\hline ichaconn1 & 196 & 32 & $\diamond$ & 6 \\
\hline ichaconn 2 & 196 & 31 & 10 & 17 \\
\hline idipigri & 157 & 32 & 188 & + \\
\hline igpp & + & 38 & 65 & + \\
\hline ihong & 11 & 22 & 16 & + \\
\hline iloadbal & 123 & 14 & 22 & $\dagger$ \\
\hline isvanberg & $\dagger$ & 27 & 48 & 53 \\
\hline antenna \iantenna2 & $\dagger$ & 186 & $\dagger$ & 156 \\
\hline antenna \iantenna_vareps & $t$ & 47 & $\dagger$ & $\dagger$ \\
\hline braess $\backslash$ itrafequil & 43 & 38 & 36 & 126 \\
\hline braess \itrafequil2 & 7 & 9 & 82 & 50 \\
\hline braess \itrafequilsf & 47 & 46 & 34 & 103 \\
\hline braess $\backslash$ itrafequil2sf & $t$ & 9 & 54 & 91 \\
\hline elena $\backslash$ ichemeq & 14 & 14 & 102 & 16 \\
\hline elena $\backslash$ is 383 & 7 & 14 & 67 & + \\
\hline firfilter $\backslash$ ifir_convex & 0 & 13 & 99 & 0 \\
\hline markowitz \igrowthopt & 15 & 16 & 22 & $\dagger$ \\
\hline wbv $\backslash$ iantenna 2 & 0 & 17 & 32 & 0 \\
\hline wbv $\backslash$ ilowpass 2 & 0 & 13 & 32 & 0 \\
\hline ibatch & 0 & 22 & 32 & 158 \\
\hline istockcycle & 117 & 35 & 72 & $\dagger$ \\
\hline isynthes 1 & 9 & 25 & 35 & 35 \\
\hline isynthes 2 & 62 & 23 & 48 & 46 \\
\hline isynthes 3 & 53 & 40 & 94 & $\dagger$ \\
\hline itrimloss 2 & 0 & 15 & 52 & 0 \\
\hline itrimloss 4 & 0 & 16 & 40 & 0 \\
\hline itrimloss 5 & 0 & 15 & 60 & 0 \\
\hline itrimloss 6 & 0 & 15 & 55 & 0 \\
\hline itrimloss7 & 0 & 16 & 61 & 0 \\
\hline itrimloss 12 & 0 & 16 & 73 & 0 \\
\hline Avg1 & 66.33 & 19.44 & - & - \\
\hline Avg2 & 11.74 & 16.04 & - & - \\
\hline
\end{tabular}

Table A.14: Comparison with MOSEK, Ipopt, and Knitro on CP infeasible test problems 